\title{
NONLOCAL PROBLEMS IN THE THEORY OF HYPERBOLIC DIFFERENTIAL EQUATIONS
}

\author{
B. PANEAH AND P. PANEAH
}

\begin{abstract}
There are relatively few local problems for general hyperbolic differential equations in a bounded domain on the plane, and all these problems are well studied, and, in simple cases, are included in almost any textbook on partial differential equations. On the contrary, nonlocal problems (even more general than boundary problems) remain practically not studied, although a number of problems of this type were successfully studied in connection with elliptic or parabolic equations. In the present paper, we consider two nonlocal quasiboundary problems of sufficiently general type in the characteristic rectangle for equations of the above type. In both cases we find conditions for unique solvability and (for the first time in the theory of hyperbolic equations) the conditions for problems to be Fredholm. Examples show that these conditions are sharp: if they are violated, the resulting problems may fail to have the required solvability properties. The proofs (in their nonanalytic part) are given in the framework of perturbation theory of operators in Banach spaces.
\end{abstract}

\section{INTRODUCTION}

In this paper we consider a strongly hyperbolic operator $\mathcal{P}$ in a bounded domain $D$ of the space $\mathbb{R}^{2}$. One of the most important problems related to this operator, which is essential from both the pure and the applied point of view, is to associate with $\mathcal{P}$ another operator $\mathcal{B}$ such that the problem

$$
\begin{cases}\mathcal{P} u=f & \text { in } D \\ \mathcal{B} u=\varphi & \text { in } \Gamma\end{cases}
$$

becomes well posed (in the sense of Hadamard). Here $\Gamma$ is, as usual, a subset in the closure $\bar{D}$ of the domain $D$ with $\operatorname{dim} \Gamma<2$. Classical examples of the problem (1) are the Cauchy problem and the Goursat problem for the operator $P$ in a characteristic triangle. Aside from the mixed problem, these are the only well-posed (and well-studied) problems for a general hyperbolic equation on the plane. A special feature of these problems is that the set $\Gamma$ lies on the boundary $\partial D$ of the domain $D$ and the value of the function $\varphi$ at each point $p \in \Gamma$ is completely determined by the value of the function $u$ and its first derivative at the same point $p$. Such problems are usually called local boundary problems. To broaden the class of problems (1), to each point $p \in \Gamma$ we associate a set $\omega_{p} \subset \bar{D}$ such that the value $\varphi(p)$ is completely determined by the values of the function $u$ on $\omega_{p}$.

Definition. Problem (1) is said to be nonlocal if $\omega_{p} \neq\{p\}$ for at least one point $p$, and is called strongly nonlocal if $\operatorname{dim} \omega_{p}>0$ for at least one point $p$.

Definition. If for some point $p$ the set $\omega_{p}$ does not lie entirely in the boundary $\partial D$, then problem (1) is said to be quasiboundary.

2000 Mathematics Subject Classification. Primary 35L57. 
The main object of study in this paper are quasiboundary nonlocal and strongly nonlocal problems, so we postpone giving examples that illustrate the introduced definitions. We only note that the problems in question were studied in the theory of elliptic and parabolic equations (see, e.g., [1, 2]). This is not the case for hyperbolic equations, even those on the plane, although some disparate attempts were made in [3. Given the role played by hyperbolic differential equations in mathematical physics, one could assume that there exist numerous nonlocal problems coming from science and engineering. However, the authors were unable to find anything in the literature. Therefore in this paper we restrict ourselves to the study of just two problems of rather general type. It turns out that both these problems are uniquely solvable in sufficiently small domains (formed by characteristics of the hyperbolic operator under consideration) independent of the coefficients of the operator $P$. Examples given in the paper show that this is the best possible result in the class of all such operators.

The next best type of solvability of problem (1) is characterized by the Fredholm property. In both problems considered in the paper, we find conditions ensuring that the corresponding problem (1) is Fredholm. These conditions turn out to be sharp: for certain operators $\mathcal{B}$ the violation of these conditions leads to infinite-dimensional kernels or cokernels of the problem.

The methods used in this paper are standard methods of the theory of linear operators in Banach spaces, combined with specific analytic tricks related to the nature of operators under consideration. The authors hope that the appearance of papers devoted to the solution of nonlocal problems for hyperbolic equations will stimulate experts in applied mathematics to explain which problems of this type are interesting in applications.

\section{Strongly NONLOCAL QUASIBOUNDARY PROBLEM}

1.1. Notation and statement of the problem. In this paper we use the following notation:

$$
I_{x}=\{x \mid 0 \leq x \leq X\}, \quad I_{y}=\{y \mid 0 \leq y \leq Y\}, \quad D=I_{x} \times I_{y}
$$

The letter $\mathcal{W}$ is used to denote the functional space

$$
\left\{w(x, y) \mid \partial_{x} w \in C(D), \partial_{y} w \in C(D), \partial_{x} \partial_{y} w \in C(D)\right\} .
$$

The norm

$$
\|w\|=|w|+\left|\partial_{x} w\right|+\left|\partial_{y} w\right|+\left|\partial_{x} \partial_{y} w\right|
$$

where

$$
|w|=\max _{(x, y) \in D}|w(x, y)|
$$

makes the linear space $\mathcal{W}$ a Banach space.

In this section we consider the following nonlocal problem (here $K_{1}(x)$ and $K_{2}(y)$ are functions integrable on intervals $I_{x}$ and $I_{y}$, respectively):

$$
\left\{\begin{array}{l}
\partial_{x} \partial_{y} u+a \partial_{x} u+b \partial_{y} u+c u=f \quad \text { in } \operatorname{int} D \\
\int_{0}^{X} K_{1}(x) u(x, y) d x=\psi(y), \quad y \in I_{y} \\
\int_{0}^{Y} K_{2}(y) u(x, y) d y=\varphi(x), \quad x \in I_{x}
\end{array}\right.
$$

Here and later we assume that the functions $a=a(x, y), b=b(x, y), c=c(x, y)$ and $f=f(x, y)$ are continuous in $D$, and the functions $\varphi(x), \psi(y)$ are differentiable on $I_{x}$ 
and $I_{y}$. Introduce the notation

$$
\kappa_{1}=\int_{0}^{X} K_{1}(x) d x, \quad \kappa_{2}=\int_{0}^{Y} K_{2}(y) d y .
$$

To the problem $(\mathcal{P})$ we associate the linear operator

$$
\mathcal{P}=\left(\mathcal{L}, \mathcal{K}_{1}, \mathcal{K}_{2}\right)
$$

acting from the space $\mathcal{W}$ to the space

$$
\mathcal{V}=C(D) \times C^{1}\left(I_{x}\right) \times C^{1}\left(I_{y}\right)
$$

where

$$
\left\{\begin{array}{l}
\mathcal{L} u(x, y)=\partial_{x} \partial_{y} u+a(x, y) \partial_{x} u+b(x, y) \partial_{y} u+c(x, y) u \\
\mathcal{K}_{1} u(y)=\int_{0}^{X} K_{1}(x) u(x, y) d x \\
\mathcal{K}_{2} u(x)=\int_{0}^{Y} K_{2}(y) u(x, y) d y .
\end{array}\right.
$$

In the operator form the problem $(\mathcal{P})$ takes the form

$$
\mathcal{P} u=(f, \varphi, \psi),
$$

and our goal is to establish invertibility properties of the operator $\mathcal{P}$.

1.2. A model problem. The problem considered in this subsection is a very special case of the general problem $(\mathcal{P})$. However, solving this problem allows us to obtain some necessary conditions for solvability of the general problem, and also to introduce the approach to this general problem in the framework of perturbation theory for bounded operators in Banach spaces. The operator $\mathcal{P}_{0}$ considered in this section plays the role of the operator "to be perturbed".

So, we consider problem $(\mathcal{P})$ in the case

$$
a(x, y)=b(x, y)=c(x, y)=0 \text { in } D,
$$

i.e., the problem

$$
\left\{\begin{array}{lr}
\partial_{x} \partial_{y} u=f & \text { in int } D \\
\int_{0}^{X} K_{1}(x) u(x, y) d x=\psi(y) & \text { on } I_{y} \\
\int_{0}^{Y} K_{2}(y) u(x, y) d y=\varphi(x) & \text { on } I_{x} .
\end{array}\right.
$$

Denote the corresponding operator $u(x, y) \rightarrow(f(x, y), \varphi(x), \psi(y))$ by $\mathcal{P}_{0}$. Multiplying the first integral condition in (2) by $\mathcal{K}_{2}(y)$ and the second integral condition by $\mathcal{K}_{1}(x)$ and integrating the resulting equations over $I_{y}$ and $I_{x}$, respectively, we see that

$$
\begin{aligned}
& \int_{0}^{X} \int_{0}^{Y} K_{2}(y) K_{1}(x) u(x, y) d y d x=\int_{0}^{X} K_{1}(x) \varphi(x) d x, \\
& \int_{0}^{X} \int_{0}^{Y} K_{2}(y) K_{1}(x) u(x, y) d y d x=\int_{0}^{Y} K_{2}(y) \psi(y) d y .
\end{aligned}
$$

This yields the first necessary solvability condition for problem (2) (hence for the general problem $(\mathcal{P})$ ):

The functions $\varphi(x)$ and $\psi(y)$ satisfy the following compatibility condition:

$$
\int_{0}^{X} K_{1}(x) \varphi(x) d x=\int_{0}^{Y} K_{2}(y) \psi(y) d y
$$


Therefore, the operator $\mathcal{P}$ (and also the operator $\mathcal{P}_{0}$ ) maps the space $\mathcal{W}$ to the space $C(D) \times F$, where

$$
F=\left\{(\varphi, \psi) \in C^{1}\left(I_{x}\right) \times C^{1}\left(I_{y}\right) \mid \int_{0}^{X} K_{1}(x) \varphi(x) d x=\int_{0}^{Y} K_{2}(y) \psi(y) d y\right\} .
$$

Let us note that the set $F$ is a closed subspace in the direct product $C^{1}\left(I_{x}\right) \times C^{1}\left(I_{y}\right)$.

Proposition 1.1. The problem (2) has a unique solution $u \in \mathcal{W}$ for each right-hand side $(f, \varphi, \psi) \in \mathcal{V}$ satisfying condition (3) if and only if $\kappa_{1} \kappa_{2} \neq 0$. Moreover, if this latter condition is satisfied, the inverse operator corresponding to problem (2) is bounded.

Proof. First we prove that under the condition $\kappa_{1} \kappa_{2} \neq 0$ the homogeneous equation $\mathcal{P}_{0} u=0$ has only the trivial solution. Next, in the case $\kappa_{1} \kappa_{2}=0$ we will present nonzero solutions of the same equation. So, let a function $u(x, y)$ be a solution of the problem

$$
\begin{aligned}
& \partial_{x} \partial_{y} u=0 \\
& \int_{0}^{X} K_{1}(x) u(x, y) d x=0, \\
& \int_{0}^{Y} K_{2}(y) u(x, y) d y=0
\end{aligned}
$$

and let $\kappa_{1} \kappa_{2} \neq 0$. Integrating (44) consecutively with respect to $y$ and to $x$ we find a general solution of this equation in the form

$$
u(x, y)=f(x)+g(y), \quad(x, y) \in D,
$$

where $f \in C^{1}\left(I_{x}\right)$ and $g \in C^{1}\left(I_{y}\right)$ are arbitrary functions. Substituting the expression (17) for $u(x, y)$ in relations (5) and (6) we obtain

$$
\left\{\begin{array}{c}
\int_{0}^{X} K_{1}(x) f(x) d x+\kappa_{1} g(y)=0 \\
\int_{0}^{Y} K_{2}(y) g(y) d y+\kappa_{2} f(x)=0 .
\end{array}\right.
$$

Introduce the notation

$$
\lambda_{1}=\lambda_{1}(f):=\int_{0}^{X} K_{1}(x) f(x) d x, \quad \lambda_{2}=\lambda_{2}(g):=\int_{0}^{Y} K_{2}(y) g(y) d y .
$$

Then a general solution of system (8) takes the form

$$
g(y)=-\frac{\lambda_{1}}{\int_{0}^{X} K_{1}(x) d x}=-\frac{\lambda_{1}}{\kappa_{1}}:=C_{1}, \quad f(x)=-\frac{\lambda_{2}}{\int_{0}^{Y} K_{2}(y) d y}=-\frac{\lambda_{2}}{\kappa_{2}}:=C_{2} .
$$

Therefore, an arbitrary solution $u(x, y)$ of the problem (4) -(6) coincides with the function $f(x)+g(y)=C_{1}+C_{2}$. In other words, $u(x, y) \equiv$ const. Substituting this value for $u(x, y)$ in (6) or in (5) and using the fact that $\kappa_{1} \kappa_{2} \neq 0$ we find that $u(x, y) \equiv 0$.

Now let $\kappa_{1} \kappa_{2}=0$. If

$$
\kappa_{1}=\kappa_{2}=0,
$$

then, clearly, an arbitrary constant function is a solution of the problem (4)-(6).

On the other hand, if

$$
\kappa_{1}=0, \quad \kappa_{2} \neq 0
$$


then for an arbitrary function $g(y) \in C_{1}\left(I_{y}\right)$, the function

$$
u(x, y)=g(y)-\frac{\int_{0}^{Y} K_{2}(y) g(y) d y}{\int_{0}^{Y} K_{2}(y) d y}
$$

is a solution of this problem, and the same is true in the case

$$
\kappa_{1} \neq 0, \quad \kappa_{2}=0 .
$$

Therefore the uniqueness in Proposition 1.1 is proved. It remains to show that problem (2) has a solution for an arbitrary right-hand side $(f(x, y), \varphi(x), \psi(y))$.

It is clear that a general solution of the differential equation in problem (2) can be represented in the form

$$
u(x, y)=\int_{0}^{x} \int_{0}^{y} f(s, t) d t d s+u_{1}(x)+u_{2}(y) .
$$

Therefore, it remains to determine functions $u_{1}(x)$ and $u_{2}(y)$ in such a way that the additional conditions in (2) were satisfied. Substituting the function $u(x, y)$ in (9) into (2) we obtain the following system of integral equations:

$$
\left\{\begin{array}{l}
\int_{0}^{X}\left[u_{1}(\xi)+u_{2}(y)+\int_{0}^{\xi} \int_{0}^{y} f(s, t) d t d s\right] K_{1}(\xi) d \xi=\psi(y) \\
\int_{0}^{Y}\left[u_{1}(x)+u_{2}(\eta)+\int_{0}^{x} \int_{0}^{\eta} f(s, t) d t d s\right] K_{2}(\eta) d \eta=\varphi(x),
\end{array}\right.
$$

or, equivalently,

$$
\left\{\begin{array}{l}
u_{2}(y) \int_{0}^{X} K_{1}(\xi) d \xi+\int_{0}^{X} K_{1}(\xi) u_{1}(\xi) d \xi+\int_{0}^{X} K_{1}(\xi) \int_{0}^{\xi} \int_{0}^{y} f(s, t) d t d s d \xi=\psi(y), \\
u_{1}(x) \int_{0}^{Y} K_{2}(\eta) d \eta+\int_{0}^{Y} K_{2}(\eta) u_{2}(\eta) d \eta+\int_{0}^{Y} K_{2}(\eta) \int_{0}^{x} \int_{0}^{\eta} f(s, t) d t d s d \eta=\varphi(x) .
\end{array}\right.
$$

This implies that

$$
\begin{aligned}
& u_{2}(y)=\frac{\psi(y)}{\kappa_{1}}-\frac{1}{\kappa_{1}} \int_{0}^{X} K_{1}(\xi) u_{1}(\xi) d \xi-\frac{1}{\kappa_{1}} \int_{0}^{X} K_{1}(\xi) \int_{0}^{\xi} \int_{0}^{y} f(s, t) d t d s d \xi \\
& u_{1}(x)=\frac{\varphi(x)}{\kappa_{2}}-\frac{1}{\kappa_{2}} \int_{0}^{Y} K_{2}(\eta) u_{2}(\eta) d \eta-\frac{1}{k_{2}} \int_{0}^{Y} K_{2}(\eta) \int_{0}^{x} \int_{0}^{\eta} f(s, t) d t d s d \eta .
\end{aligned}
$$

Adding together both relations in (10), we see that

$$
\begin{aligned}
u_{1}(x)+u_{2}(y)= & \frac{\varphi(x)}{\kappa_{2}}+\frac{\psi(y)}{\kappa_{1}}-\frac{1}{\kappa_{1}} \int_{0}^{X} K_{1}(\xi) \int_{0}^{\xi} \int_{0}^{y} f(s, t) d t d s d \xi \\
& -\frac{1}{k_{2}} \int_{0}^{Y} K_{2}(\eta) \int_{0}^{x} \int_{0}^{\eta} f(s, t) d t d s d \eta \\
& -\frac{1}{\kappa_{1}} \int_{0}^{X} K_{1}(\xi) u_{1}(\xi) d \xi-\frac{1}{\kappa_{2}} \int_{0}^{Y} K_{2}(\eta) u_{2}(\eta) d \eta .
\end{aligned}
$$

To eliminate the terms

$$
\int_{0}^{X} u_{1}(\xi) K_{1}(\xi) d \xi \quad \text { and } \quad \int_{0}^{Y} u_{2}(\eta) K_{2}(\eta) d \eta
$$


in (11) we multiply the first relation in (10) by $K_{2}(y) / \kappa_{2}$ and integrate the product over the interval $I_{y}$. This yields the relation

$$
\begin{aligned}
\frac{1}{\kappa_{2}} \int_{0}^{Y} K_{2}(y) u_{2}(y) d y= & \frac{1}{\kappa_{1} \kappa_{2}} \int_{0}^{Y} K_{2}(y) \psi(y) d y-\frac{1}{\kappa_{1} \kappa_{2}} \int_{0}^{Y} K_{2}(y) \int_{0}^{X} K_{1}(\xi) u_{1}(\xi) d \xi d y \\
& -\frac{1}{\kappa_{1} \kappa_{2}} \int_{0}^{Y} K_{2}(y) \int_{0}^{X} K_{1}(\xi) \int_{0}^{\xi} \int_{0}^{y} f(s, t) d t d s d \xi d y
\end{aligned}
$$

which implies that

$$
\begin{aligned}
& \frac{1}{\kappa_{2}} \int_{0}^{Y} K_{2}(y) u_{2}(y) d y+\frac{1}{\kappa_{1}} \int_{0}^{X} K_{1}(\xi) u_{1}(\xi) d \xi \\
& \quad=\frac{1}{\kappa_{1} \kappa_{2}} \int_{0}^{Y} K_{2}(y) \psi(y) d y-\frac{1}{\kappa_{1} \kappa_{2}} \int_{0}^{X} \int_{0}^{Y} K_{1}(\xi) K_{2}(y) \int_{0}^{\xi} \int_{0}^{y} f(s, t) d t d s d y d \xi
\end{aligned}
$$

Combining relations (111) and (12) we see that

$$
\begin{aligned}
u_{1}(x)+u_{2}(y)= & \frac{\varphi(x)}{\kappa_{2}}+\frac{\psi(y)}{\kappa_{1}}-\frac{1}{\kappa_{1}} \int_{0}^{X} K_{1}(\xi) \int_{0}^{\xi} \int_{0}^{y} f(s, t) d t d s d \xi \\
& -\frac{1}{\kappa_{2}} \int_{0}^{Y} K_{2}(\eta) \int_{0}^{x} \int_{0}^{\eta} f(s, t) d t d s d \eta \\
& +\frac{1}{\kappa_{1} \kappa_{2}} \int_{0}^{X} \int_{0}^{Y} K_{1}(\xi) K_{2}(\eta) \int_{0}^{\xi} \int_{0}^{\eta} f(s, t) d t d s d \eta d \xi \\
& -\frac{1}{\kappa_{1} \kappa_{2}} \int_{0}^{Y} K_{2}(\eta) \psi(\eta) d \eta .
\end{aligned}
$$

Substituting the obtained expression for $u_{1}(x)+u_{2}(y)$ in (9) we get the function

$$
\begin{aligned}
u(x, y)= & \int_{0}^{x} \int_{0}^{y} f(s, t) d t d s-\frac{1}{\kappa_{1}} \int_{0}^{X} K_{1}(\xi)\left(\int_{0}^{\xi} \int_{0}^{y} f(s, t) d t d s\right) d \xi \\
& -\frac{1}{\kappa_{2}} \int_{0}^{Y} K_{2}(\eta)\left(\int_{0}^{x} \int_{0}^{\eta} f(s, t) d t d s\right) d \eta \\
& +\frac{1}{\kappa_{1} \kappa_{2}} \int_{0}^{X} \int_{0}^{Y} K_{1}(\xi) K_{2}(\eta)\left(\int_{0}^{\xi} \int_{0}^{\eta} f(s, t) d t d s\right) d \eta d \xi \\
& -\frac{1}{\kappa_{1} \kappa_{2}} \int_{0}^{Y} K_{2}(\eta) \psi(\eta) d \eta+\frac{\varphi(x)}{\kappa_{2}}+\frac{\psi(y)}{\kappa_{1}}
\end{aligned}
$$

representing the unique solution of problem (2). One can verify directly that this solution belongs to the space $\mathcal{W}$ and that the operator $(f, \varphi, \psi) \in \mathcal{V} \rightarrow u \in \mathcal{W}$ is continuous. Hence Proposition 1.1 is proved.

Remark 1. Using the compatibility conditions (3), we can represent the function

$$
u=\mathcal{P}_{0}^{-1}(f, \varphi, \psi)
$$


in the form symmetric with respect to the variables $x$ and $y$, namely,

$$
\begin{aligned}
u(x, y)= & \int_{0}^{x} \int_{0}^{y} f(s, t) d t d s-\frac{1}{\kappa_{1}} \int_{0}^{X} K_{1}(\xi)\left(\int_{0}^{\xi} \int_{0}^{y} f(s, t) d t d s\right) d \xi \\
& -\frac{1}{\kappa_{2}} \int_{0}^{Y} K_{2}(\eta)\left(\int_{0}^{x} \int_{0}^{\eta} f(s, t) d t d s\right) d \eta \\
& +\frac{1}{\kappa_{1} \kappa_{2}} \int_{0}^{\alpha} \int_{0}^{\beta} K_{1}(\xi) K_{2}(\eta)\left(\int_{0}^{\xi} \int_{0}^{\eta} f(s, t) d t d s\right) d \eta d \xi \\
& -\frac{1}{2 \kappa_{1} \kappa_{2}}\left(\int_{0}^{Y} K_{2}(\eta) \psi(\eta) d \eta+\int_{0}^{X} K_{1}(\xi) \phi(\xi) d \xi\right)+\frac{\varphi(x)}{\kappa_{2}}+\frac{\psi(y)}{\kappa_{1}}
\end{aligned}
$$

1.3. Reduction of problem $(\mathcal{P})$ to an integral equation. In this subsection we show how to reduce problem $(\mathcal{P})$ to an equivalent integral equation. This will allow us to use the methods of functional analysis.

Introduce the following integral operator:

$$
\begin{aligned}
\mathcal{C}: v(x, y) \mapsto v(x, y)+ & a(x, y)\left[\int_{0}^{y} v(x, t) d t-\frac{1}{\kappa_{2}} \int_{0}^{Y} K_{2}(\eta) \int_{0}^{\eta} v(x, t) d t d \eta\right] \\
+b(x, y)\left[\int_{0}^{x} v(s, y) d s-\frac{1}{\kappa_{1}} \int_{0}^{X} K_{1}(\xi) \int_{0}^{\xi} v(s, y) d s d \xi\right] & {\left[\int_{0}^{x} \int_{0}^{y} v(s, t) d t d s-\frac{1}{\kappa_{1}} \int_{0}^{X} K_{1}(\xi) \int_{0}^{\xi} \int_{0}^{y} v(s, t) d t d s d \xi\right.} \\
+c(x, y) & -\frac{1}{\kappa_{2}} \int_{0}^{Y} K_{2}(\eta) \int_{0}^{x} \int_{0}^{\eta} v(s, t) d t d s d \eta \\
& \left.+\frac{1}{\kappa_{1} \kappa_{2}} \int_{0}^{X} \int_{0}^{Y} K_{1}(\xi) K_{2}(\eta) \int_{0}^{\xi} \int_{0}^{\eta} v(s, t) d t d s d \eta d \xi\right]
\end{aligned}
$$

in the space $C(D)$ and associate to it the integral equation

$$
\begin{aligned}
\mathcal{C} v(x, y)= & f(x, y)-\frac{a(x, y)}{\kappa_{2}} \varphi^{\prime}(x)-\frac{b(x, y)}{\kappa_{1}} \psi^{\prime}(y) \\
& +c(x, y)\left(\frac{1}{\kappa_{1} \kappa_{2}} \int_{0}^{Y} K_{2}(\eta) \psi(\eta) d \eta-\frac{1}{\kappa_{2}} \varphi(x)-\frac{1}{\kappa_{1}} \psi(y)\right) .
\end{aligned}
$$

Theorem 1.2. If $\kappa_{1} \kappa_{2} \neq 0$, then problem $(\mathcal{P})$ has a solution $u \in \mathcal{W}$ if and only if equation (13) is solvable in the space $C(D)$. This solution can be represented in the form

$$
u(x, y)=\mathcal{P}_{0}^{-1}(v(x, y), \varphi(x), \psi(y))
$$

where $v(x, y)$ is the solution of equation (13). 
Proof. A direct verification shows that if a function $u(x, y)$ is given by (14) with $v(x, y) \in$ $C(D)$, then $u \in \mathcal{W}$. For each such function $u(x, y)$ we have

$$
\begin{aligned}
& \partial_{x} \partial_{y}\left[\int_{0}^{x} \int_{0}^{y} v(s, t) d t d s-\frac{1}{\kappa_{1}} \int_{0}^{X} K_{1}(\xi) \int_{0}^{\xi} \int_{0}^{y} v(s, t) d t d s d \xi\right. \\
& -\frac{1}{\kappa_{2}} \int_{0}^{Y} K_{2}(\eta) \int_{0}^{x} \int_{0}^{\eta} v(s, t) d t d s d \eta-\frac{1}{\kappa_{1} \kappa_{2}} \int_{0}^{Y} K_{2}(\eta) \psi(\eta) d \eta \\
& \left.+\frac{1}{\kappa_{1} \kappa_{2}} \int_{0}^{X} \int_{0}^{Y} K_{1}(\xi) K_{2}(y) \int_{0}^{\xi} \int_{0}^{\eta} v(s, t) d t d s d \eta d \xi+\frac{\varphi(x)}{\kappa_{2}}+\frac{\psi(y)}{\kappa_{1}}\right] \\
& +a(x, y) \partial_{x}\left[\int_{0}^{x} \int_{0}^{y} v(s, t) d t d s-\frac{1}{\kappa_{1}} \int_{0}^{X} K_{1}(\xi) \int_{0}^{\xi} \int_{0}^{y} v(s, t) d t d s d \xi\right. \\
& -\frac{1}{\kappa_{2}} \int_{0}^{Y} K_{2}(\eta) \int_{0}^{x} \int_{0}^{\eta} v(s, t) d t d s d \eta-\frac{1}{\kappa_{1} \kappa_{2}} \int_{0}^{Y} K_{2}(\eta) \psi(\eta) d \eta \\
& \left.+\frac{1}{\kappa_{1} \kappa_{2}} \int_{0}^{X} \int_{0}^{Y} K_{1}(\xi) K_{2}(y) \int_{0}^{\xi} \int_{0}^{y} v(s, t) d t d s d y d \xi+\frac{\varphi(x)}{\kappa_{2}}+\frac{\psi(y)}{\kappa_{1}}\right] \\
& +b(x, y) \partial y\left[\int_{0}^{x} \int_{0}^{y} v(s, t) d t d s-\frac{1}{\kappa_{1}} \int_{0}^{X} K_{1}(\xi) \int_{0}^{\xi} \int_{0}^{y} v(s, t) d t d s d \xi\right. \\
& -\frac{1}{\kappa_{2}} \int_{0}^{Y} K_{2}(\eta) \int_{0}^{x} \int_{0}^{\eta} v(s, t) d t d s d \eta-\frac{1}{\kappa_{1} \kappa_{2}} \int_{0}^{Y} K_{2}(\eta) \psi(\eta) d \eta \\
& \left.+\frac{1}{\kappa_{1} \kappa_{2}} \int_{0}^{X} \int_{0}^{Y} K_{1}(\xi) K_{2}(\eta) \int_{0}^{\xi} \int_{0}^{\eta} v(s, t) d t d s d \eta d \xi+\frac{\varphi(x)}{\kappa_{2}}+\frac{\psi(y)}{\kappa_{1}}\right] \\
& +c(x, y)\left[\int_{0}^{x} \int_{0}^{y} v(s, t) d t d s-\frac{1}{\kappa_{1}} \int_{0}^{X} K_{1}(\xi) \int_{0}^{\xi} \int_{0}^{y} v(s, t) d t d s d \xi\right. \\
& -\frac{1}{\kappa_{2}} \int_{0}^{Y} K_{2}(\eta) \int_{0}^{x} \int_{0}^{\eta} v(s, t) d t d s d \eta-\frac{1}{\kappa_{1} \kappa_{2}} \int_{0}^{Y} K_{2}(\eta) \psi(\eta) d \eta \\
& \left.+\frac{1}{\kappa_{1} \kappa_{2}} \int_{0}^{X} \int_{0}^{Y} K_{1}(\xi) K_{2}(y) \int_{0}^{\xi} \int_{0}^{y} v(s, t) d t d s d y d \xi+\frac{\varphi(x)}{\kappa_{2}}+\frac{\psi(y)}{\kappa_{1}}\right] \\
& =f(x, y) \text {. }
\end{aligned}
$$

Again by direct verification one sees that this relation coincides with (13), thus proving the necessity part of the theorem.

To prove sufficiency we verify that if a function $v(x, y)$ is a solution of equation (13), then the function $u(x, y)$ defined as the solution of the problem $\mathcal{P}_{0} u=(v, \varphi, \psi)$ satisfies the equation $\mathcal{P} u=(f, \varphi, \psi)$. Indeed, the definition of the operator $\mathcal{P}_{0}$ (see (2)) implies that

$$
v=\partial_{x} \partial_{y} u,
$$

and

$$
\begin{aligned}
& \psi(y)=\int_{0}^{X} K_{1}(x) u(x, y) d x, \\
& \varphi(x)=\int_{0}^{Y} K_{2}(y) u(x, y) d y .
\end{aligned}
$$


Substituting $\partial_{x} \partial_{y} u$ instead of $\partial_{x} \partial_{y} u$ in (13) and using (15) and (16), we find that

$$
\begin{aligned}
& \partial_{x} \partial_{y} u+a(x, y)\left\{\partial_{x} u(x, y)-\partial_{x} u(x, 0)-\frac{1}{\kappa_{2}} \int_{0}^{Y} K_{2}(\eta)\left[\partial_{x} u(x, \eta)-\partial_{x} u(x, 0)\right] d \eta\right\} \\
&+b(x, y)\left\{\partial_{y} u(x, y)-\partial_{y} u(0, y)-\frac{1}{\kappa_{1}} \int_{0}^{X} K_{1}(\xi)\left[\partial_{y} u(\xi, y)-\partial_{y} u(0, y)\right] d \xi\right\} \\
&+c(x, y)\{u(x, y)-u(0, y)-u(x, 0)+u(0,0) \\
&-\frac{1}{\kappa_{1}} \int_{0}^{X} K_{1}(\xi)[u(\xi, y)-u(0, y)-u(\xi, 0)+u(0,0)] d \xi \\
&-\frac{1}{\kappa_{2}} \int_{0}^{Y} K_{2}(\eta)[u(x, \eta)-u(0, \eta)-u(x, 0)+u(0,0)] d \eta \\
&\left.+\frac{1}{\kappa_{1} \kappa_{2}} \int_{0}^{X} \int_{0}^{Y} K_{1}(\xi) K_{2}(\eta)[u(\xi, \eta)-u(0, \eta)-u(\xi, 0)+u(0,0)] d \eta d \xi\right\} \\
&= \partial_{x} \partial_{y} u+a(x, y)\left[\partial_{x} u(x, y)-\frac{1}{\kappa_{2}} \varphi^{\prime}(x)\right]+b(x, y)\left[\partial_{y} u(x, y)-\frac{1}{\kappa_{1}} \psi^{\prime}(y)\right] \\
&+c(x, y)\left[u(x, y)-\frac{1}{\kappa_{1}} \psi(y)-\frac{1}{\kappa_{2}} \varphi(x)+\frac{1}{\kappa_{1} \kappa_{2}} \int_{0}^{Y} K_{2}(\eta) \psi(\eta) d \eta\right] .
\end{aligned}
$$

Together with (13) this implies that

$$
\partial_{x} \partial_{y} u+a u_{x}+b u_{y}+c u=f,
$$

which, together with (15) and (16), shows that the function $u(x, y)$ solves problem $(\mathcal{P})$. Theorem 1.2 is completely proved.

1.4. Unique solvability of problem $(\mathcal{P})$. In this subsection we show that under certain conditions on the functions $K_{1}$ and $K_{2}$, problem $(\mathcal{P})$ has a unique solution for each right-hand side $(f, \varphi, \psi)$ satisfying the necessary condition (3). Moreover, we will show that if these conditions on $K_{1}$ and $K_{2}$ are not satisfied, problem $(\mathcal{P})$ can have multiple solutions or even no solutions at all.

Introduce the following notation:

$$
\begin{aligned}
\bar{\kappa}_{1} & :=\frac{1}{X\left|\kappa_{1}\right|} \int_{0}^{X} x\left|K_{1}(x)\right| d x, \\
\bar{\kappa}_{2} & :=\frac{1}{Y\left|\kappa_{2}\right|} \int_{0}^{Y} y\left|K_{2}(y)\right| d y, \\
\bar{\kappa} & :=\max \left(\bar{\kappa}_{1}, \bar{\kappa}_{2}, \bar{\kappa}_{1} \bar{\kappa}_{2}\right), \\
\gamma & :=\max (X|b|, Y|a|, X Y|c|) .
\end{aligned}
$$

Theorem 1.3. If $\kappa_{1} \kappa_{2} \neq 0$ and functions $K_{1}(x), K_{2}(y)$ satisfy the condition

$$
\bar{\kappa}<\frac{e^{-9 \gamma}}{5 \gamma}
$$

then for any functions $(f(x, y), \varphi(x), \psi(y)) \in \mathcal{V}$ satisfying the necessary condition (3), problem $(\mathcal{P})$ has a unique solution. 
Proof. Consider problem $(\mathcal{P})$ for given $f(x, y), \varphi(x), \psi(y)$ and introduce the function

$$
\begin{aligned}
\tilde{f}(x, y):= & f(x, y)-\frac{a(x, y)}{\kappa_{2}} \varphi^{\prime}(x)-\frac{b(x, y)}{\kappa_{1}} \psi^{\prime}(y) \\
& +\frac{c(x, y)}{\kappa_{1} \kappa_{2}} \int_{0}^{Y} K_{2}(y) \psi(y) d y-\frac{c(x, y)}{\kappa_{2}} \varphi(x)-\frac{c(x, y)}{\kappa_{1}} \psi(y),
\end{aligned}
$$

which appears in the integral equation (13).

Introduce also two new operators

$$
\begin{aligned}
(A v)(x, y):= & a(x, y) \int_{0}^{y} v(x, t) d t \\
& +b(x, y) \int_{0}^{x} v(s, y) d s+c(x, y) \int_{0}^{x} \int_{0}^{y} v(s, t) d t d s \text { in } D
\end{aligned}
$$

and

$$
\begin{aligned}
(B v)(x, y):= & -\frac{a(x, y)}{\kappa_{2}} \int_{0}^{Y} K_{2}(\eta) \int_{0}^{\eta} v(x, t) d t d \eta \\
& -\frac{b(x, y)}{\kappa_{1}} \int_{0}^{X} K_{1}(\xi) \int_{0}^{\xi} v(s, y) d s d \xi \\
& -\frac{c(x, y)}{\kappa_{1}} \int_{0}^{X} K_{1}(\xi) \int_{0}^{\xi} \int_{0}^{y} v(s, t) d t d s d \xi \\
& -\frac{c(x, y)}{\kappa_{2}} \int_{0}^{Y} K_{2}(\eta) \int_{0}^{x} \int_{0}^{\eta} v(s, t) d t d s d \eta \\
& +\frac{c(x, y)}{\kappa_{1} \kappa_{2}} \int_{0}^{X} \int_{0}^{Y} K_{1}(\xi) K_{2}(\eta) \int_{0}^{\xi} \int_{0}^{\eta} v(s, t) d t d s d \eta d \xi \text { in } D .
\end{aligned}
$$

The integral equation (13) corresponding to problem $(\mathcal{P})$ can now be rewritten in the following operator form:

$$
(I+A) v+B v=\widetilde{f} .
$$

Assume that we have proved the invertibility of the operator $I+A$ in the space $C(D)$ and obtained the estimate

$$
\left\|(I+A)^{-1} B\right\|<1 .
$$

Then unique solvability of equation (19) for an arbitrary right-hand side $f$ easily follows from a well-known theorem of functional analysis. Taking into account Theorem 1.2 we see that solvability of equation (19) implies the existence of a solution $u(x, y)$ of problem $(\mathcal{P})$ for all functions $f(x, y), \varphi(x), \psi(y)$ in the corresponding spaces. Let us verify the uniqueness of such a solution. To do this let us return to problem $(\mathcal{P})$ with $f(x, y)=0$, $\varphi(x)=0, \psi(y)=0$. Let $u(x, y)$ be one of the solutions of this problem. Then, as was proved earlier, the function

$$
v(x, y)=\partial_{x} \partial_{y} u(x, y)
$$

is a solution of the problem

$$
v(x, y)+(I+A)^{-1} B v(x, y)=0
$$

and according to (20),

$$
v(x, y)=0 .
$$

This means that for certain functions

$$
\alpha(x) \in C^{1}\left(I_{x}\right) \quad \text { and } \quad \beta(y) \in C^{1}\left(I_{y}\right)
$$


we have

$$
u(x, y)=\alpha(x)+\beta(y) .
$$

But the formulation of problem $(\mathcal{P})$ shows that

$$
\begin{aligned}
& \int_{0}^{X} \alpha(x) K_{1}(x) d x+\left(\int_{0}^{X} K_{1}(x) d x\right) \beta(y)=0 \\
& \int_{0}^{Y} \beta(y) K_{2}(y) d y+\left(\int_{0}^{Y} K_{2}(y) d y\right) \alpha(x)=0 .
\end{aligned}
$$

This means that both functions $\alpha(x)$ and $\beta(y)$ are constants $\alpha$ and $\beta$, respectively, and that these constants satisfy the conditions

$$
\begin{gathered}
\alpha \kappa_{1}+\kappa_{1} \beta=0, \\
\beta \kappa_{2}+\kappa_{2} \alpha=0 .
\end{gathered}
$$

Since $\kappa_{1} \kappa_{2} \neq 0$ this implies that

$$
\alpha+\beta=0
$$

hence also

$$
u(x, y)=0 .
$$

Thus the required uniqueness of the solution of problem $(\mathcal{P})$ is established, and the proof of Theorem 1.3 is completed. Hence it remains to prove the invertibility of the operator $I+A$ and to establish estimate (20). The first of these facts is an easy consequence of the following result.

Lemma 1.4. For each operator $A$ of the form (18) we have

$$
\sum_{n=0}^{\infty}\left\|A^{n}\right\|<e^{9 \gamma}
$$

where $\|\cdot\|$ is the standard norm in the space of linear operators acting in the space $C(D)$.

Proof. Introduce the operators

$$
\begin{aligned}
& \left(A_{1} v\right)(x, y):=a(x, y) \int_{0}^{y} v(x, t) d t \quad \text { in } D, \\
& \left(A_{2} v\right)(x, y):=b(x, y) \int_{0}^{x} v(s, y) d s \text { in } D, \\
& \left(A_{3} v\right)(x, y):=c(x, y) \int_{0}^{x} \int_{0}^{y} v(s, t) d t d s \text { in } D
\end{aligned}
$$

and the operators

$$
\begin{aligned}
& \widetilde{A}_{1} v(x, y)=|a| \int_{0}^{y}|v(x, t)| d t \quad \text { in } D, \\
& \widetilde{A}_{2} v(x, y)=|b| \int_{0}^{x}|v(s, y)| d s \text { in } D, \\
& \widetilde{A}_{3} v(x, y)=|c| \int_{0}^{x} \int_{0}^{y}|v(s, t)| d t d s \text { in } D .
\end{aligned}
$$

Let us prove that the inequality

$$
\left|\left(A_{1}^{n} v\right)(x, y)\right| \leq\left(\widetilde{A}_{1}^{n} v\right)(x, y)
$$

holds at each point $(x, y) \in D$. Indeed, for $n=1$ we have

$$
\left|\left(A_{1} v\right)(x, y)\right|=|a(x, y)|\left|\int_{0}^{y} v(x, t) d t\right| \leq\left(\widetilde{A}_{1} v\right)(x, y) \quad \text { in } D \text {. }
$$


Let us assume that for some $k \geq 1$ we have

$$
\left|\left(A_{1}^{k} v\right)(x, y)\right| \leq\left(\widetilde{A}_{1}^{k} v\right)(x, y) \text { in } D
$$

and show that

$$
\left|\left(A_{1}^{k+1} v\right)(x, y)\right| \leq\left(\widetilde{A}_{1}^{k+1} v\right)(x, y) \text { in } D
$$

Indeed,

$$
\begin{aligned}
\left|\left(A_{1}^{k+1} v\right)(x, y)\right| & =\left|\left(A_{1}\left(A_{1}^{k} v\right)\right)(x, y)\right| \\
& =|a(x, y)|\left|\int_{0}^{y}\left(A_{1}^{k} v\right)(x, t) d t\right| \leq|a| \int_{0}^{y}\left|\left(A_{1}^{k} v\right)(x, t)\right| d t .
\end{aligned}
$$

Inequality (22) shows that

$$
|a| \int_{0}^{y}\left|\left(A_{1}^{k} v\right)(x, t)\right| d t \leq|a| \int_{0}^{y}\left(\widetilde{A}_{1}^{k} v\right)(x, t) d t=\left(\widetilde{A}_{1}^{k+1} v\right)(x, y) .
$$

Therefore, the required relation (21) holds for all $n \in \mathbb{N}$.

To simplify the notation, introduce the unit sphere

$$
S=\{v(x, y)|| v \mid=1\} .
$$

Using induction, let us prove that for each $n \in \mathbb{N}$ and for an arbitrary function $v(x, y) \in S$, we have

$$
\left(\widetilde{A}_{1}^{n} v\right)(x, y) \leq|a|^{n} \frac{y^{n}}{n !} \quad \text { in } D .
$$

Indeed, for $n=1$ we have

$$
\left(\widetilde{A}_{1} v\right)(x, y)=|a| \int_{0}^{y}|v(x, t)| d t \leq|a| \int_{0}^{y} d t=|a| y .
$$

Let us assume that for some $k \geq 1$ we have

$$
\left(\widetilde{A}_{1}^{k} v\right)(x, y) \leq|a|^{k} \frac{y^{k}}{k !} \quad \text { in } D .
$$

By the induction hypothesis,

$$
\begin{aligned}
\left(\widetilde{A}_{1}^{k+1} v\right)(x, y)=\left(\widetilde{A}_{1}\left(\widetilde{A}_{1}^{k} v\right)\right)(x, y) & =|a| \int_{0}^{y}\left|\left(\widetilde{A}_{1}^{k} v\right)(x, t)\right| d t \\
& \leq|a| \int_{0}^{y}|a|^{k} \frac{t^{k}}{k !} d t=|a|^{k+1} \frac{y^{k+1}}{(k+1) !} .
\end{aligned}
$$

Therefore, (23) holds for all $n \in \mathbb{N}$.

Together with (23), inequality (21) allows us to conclude that for each function $v \in S$, the inequality

$$
\left|\left(A_{1}^{n} v\right)(x, y)\right| \leq\left(\widetilde{A}_{1}^{n} v\right)(x, y) \leq|a|^{n} \frac{y^{n}}{n !} \quad \text { in } D
$$

holds at all points $(x, y) \in D$.

A similar estimate for the operator $A_{2}$, namely

$$
\left|\left(A_{2}^{n} v\right)(x, y)\right| \leq\left(\widetilde{A}_{2}^{n} v\right)(x, y) \leq|b|^{n} \frac{x^{n}}{n !} \quad \text { in } D,
$$

takes place for all functions $v \in S$ defined in $D$.

Repeating the above arguments for the operator $A_{3}$ we conclude that for all functions $v(x, y) \in S$, the following estimate holds at each point $(x, y) \in D$ :

$$
\left|\left(A_{3}^{n} v\right)(x, y)\right| \leq\left(\widetilde{A}_{3}^{n} v\right)(x, y) \leq|c|^{n} \frac{(x y)^{n}}{(n !)^{2}} \quad \text { in } D .
$$


It is important to note that the above operators $\widetilde{A}_{1}, \widetilde{A}_{2}$, and $\widetilde{A}_{3}$ pairwise commute. Indeed, consider, for example, the pair of operators $\widetilde{A}_{1}$ and $\widetilde{A}_{2}$. Then for each function $v \in C(D)$ we have

$$
\begin{aligned}
& \left(\widetilde{A}_{1}\left(\widetilde{A}_{2} v\right)\right)(x, y)=|a| \int_{0}^{y}|| b\left|\int_{0}^{x}\right| v(s, t)|d s| d t=|a||b| \int_{0}^{y} \int_{0}^{x}|v(s, t)| d s d t \quad \text { in } D, \\
& \left(\widetilde{A}_{2}\left(\widetilde{A}_{1} v\right)\right)(x, y)=|b| \int_{0}^{x}|| a\left|\int_{0}^{y}\right| v(s, t)|d t| d s=|b||a| \int_{0}^{x} \int_{0}^{y}|v(s, t)| d t d s \text { in } D .
\end{aligned}
$$

By the Fubini theorem, this implies the required commutativity of the operators $\widetilde{A}_{1}$ and $\widetilde{A}_{2}$. For operators $\widetilde{A}_{1}$ and $\widetilde{A}_{3}$ the same arguments using the Fubini theorem yield the equality $\widetilde{A}_{1} \widetilde{A}_{3}=\widetilde{A}_{3} \widetilde{A}_{1}$. Our next step is the verification of the formula

$$
\left|\left(\left(A_{1}+A_{2}+A_{3}\right)^{n} v\right)(x, y)\right| \leq\left(\left(\widetilde{A}_{1}+\widetilde{A}_{2}+\widetilde{A}_{3}\right)^{n} v\right)(x, y) \text { in } D .
$$

For $n=1$ we use standard estimates of integrals to find that

$$
\begin{aligned}
\left|\left(\left(A_{1}+A_{2}+A_{3}\right) v\right)(x, y)\right| & =\left|\left(A_{1} v\right)(x, y)+\left(A_{2} v\right)(x, y)+\left(A_{3} v\right)(x, y)\right| \\
& \leq\left|\left(A_{1} v\right)(x, y)\right|+\left|\left(A_{2} v\right)(x, y)\right|+\left|\left(A_{3} v\right)(x, y)\right| \\
& \leq\left(\widetilde{A}_{1} v\right)(x, y)+\left(\widetilde{A}_{2} v\right)(x, y)+\left(\widetilde{A}_{3} v\right)(x, y) \\
& =\left(\left(\widetilde{A}_{1}+\widetilde{A}_{2}+\widetilde{A}_{3}\right) v\right)(x, y) .
\end{aligned}
$$

Let us show that this formula and inequality (27) for an arbitrary $k \in \mathbb{N}$ imply the same inequality for $k+1$. Introduce the notation

$$
w(x, y):=\left(\left(A_{1}+A_{2}+A_{3}\right)^{k} v\right)(x, y) .
$$

Then (28) implies that

$$
\begin{aligned}
\left|\left(\left(A_{1}+A_{2}+A_{3}\right)^{k+1} v\right)(x, y)\right| & =\left|\left(\left(A_{1}+A_{2}+A_{3}\right) w\right)(x, y)\right| \\
& \leq\left(\left(\widetilde{A}_{1}+\widetilde{A}_{2}+\widetilde{A}_{3}\right) w\right)(x, y) .
\end{aligned}
$$

Let us note that the definition of the operators $A_{j}, j=1,2,3, \ldots$, shows that the inequality

$$
0 \leq u_{1}(x, y) \leq u_{2}(x, y) \text { in } D
$$

for functions $u_{1}, u_{2}$ in the space $C(D)$ implies that

$$
\left(\widetilde{A}_{j} u_{1}\right)(x, y) \leq\left(\widetilde{A}_{j} u_{2}\right)(x, y) \text { in } D .
$$

Together with the induction hypothesis, these arguments yield the inequality

$$
\begin{aligned}
& \left(\left(\widetilde{A}_{1}+\widetilde{A}_{2}+\widetilde{A}_{3}\right) w\right)(x, y)=\left(\left(\widetilde{A}_{1}+\widetilde{A}_{2}+\widetilde{A}_{3}\right)\left(A_{1}+A_{2}+A_{3}\right)^{k} v\right)(x, y) \\
& \quad=\left(\left(\widetilde{A}_{1}+\widetilde{A}_{2}+\widetilde{A}_{3}\right)\left|\left(A_{1}+A_{2}+A_{3}\right)^{k} v\right|\right)(x, y) \\
& \quad \leq\left(\left(\widetilde{A}_{1}+\widetilde{A}_{2}+\widetilde{A}_{3}\right)\left(\widetilde{A}_{1}+\widetilde{A}_{2}+\widetilde{A}_{3}\right)^{k} v\right)(x, y)=\left(\left(\widetilde{A}_{1}+\widetilde{A}_{2}+\widetilde{A}_{3}\right)^{k+1} v\right)(x, y) .
\end{aligned}
$$

In turn, the latter inequality and (29) together imply the required relation (27) for each $n \in \mathbb{N}$.

Using (27) and the commutativity of the operators $\widetilde{A}_{1}, \widetilde{A}_{2}$, and $\widetilde{A}_{3}$, we have the following inequality for all functions $v \in C(D)$ :

$$
\begin{aligned}
\left|\left(\left(A_{1}+A_{2}+A_{3}\right)^{n} v\right)(x, y)\right| & \leq\left(\left(\widetilde{A}_{1}+\widetilde{A}_{2}+\widetilde{A}_{3}\right)^{n} v\right)(x, y) \\
& =\left(\left(\sum_{\substack{0 \leq i, j, k \leq n \\
i+j+k=n}} \frac{n !}{i ! j ! k !} \widetilde{A}_{1}^{i} \widetilde{A}_{2}^{j} \widetilde{A}_{3}^{k}\right) v\right)(x, y) .
\end{aligned}
$$


Now we estimate from above the right-hand side of the latter inequality for functions $v \in S$. It is clear that relation (30) remains true for all integer powers $\widetilde{A}_{j}^{m}$ of operators $\widetilde{A}_{j}, 1 \leq j \leq 3$, and for all products of these powers. This comment, together with estimates (24), (25), (26) yields the following estimate:

$$
\begin{aligned}
&\left(\left(\sum_{\substack{0 \leq i, j, k \leq n \\
i+j+k=n}} \frac{n !}{i ! j ! k !} \widetilde{A}_{1}^{i} \widetilde{A}_{2}^{j} \widetilde{A}_{3}^{k}\right) v\right)(x, y) \\
& \quad \leq\left(\sum_{\substack{0 \leq i, j, k \leq n \\
i+j+k=n}} \frac{n !}{i ! j ! k !} \widetilde{A}_{1}^{i} \widetilde{A}_{2}^{j}\right) \frac{(|c| X Y)^{k}}{(k !)^{2}} \\
& \quad=\left(\sum_{\substack{0 \leq i, j, k \leq n \\
i+j+k=n}} \frac{n !}{i ! j ! k !} \frac{(|c| X Y)^{k}}{(k !)^{2}} \widetilde{A}_{1}^{i} \widetilde{A}_{2}^{j}\right)(1) \\
& \leq\left(\sum_{\substack{0 \leq i, j, k \leq n \\
i+j+k=n}} \frac{n !}{i ! j ! k !} \frac{(|c| X Y)^{k}}{(k !)^{2}} \widetilde{A}_{1}^{i}\right)\left(\frac{(|b| X)^{j}}{j !}\right) \\
&=\left(\sum_{\substack{0 \leq i, j, k \leq n \\
i+j+k=n}} \frac{n !}{i ! j ! k !} \frac{(|c| X Y)^{k}}{(k !)^{2}} \frac{(|b| X)^{j}}{j !} \widetilde{A}_{1}^{i}\right)(1) \\
& \leq \sum_{\substack{0 \leq i, j, k \leq n \\
i+j+k=n}} \frac{n !}{i ! j ! k !} \frac{(|c| X Y)^{k}}{(k !)^{2}} \frac{(|b| X)^{j}}{j !} \frac{(|a| Y)^{i}}{i !} \\
& \leq \gamma^{n} \sum_{\substack{0 \leq i, j, k \leq n \\
i+j+k=n}} \frac{n !}{(i ! j !)^{2}(k !)^{3}} \leq \frac{\gamma^{n}}{n !} \sum_{\substack{0 \leq i, j, k \leq n \\
i+j+k=n}} \frac{(n !)^{2}}{(i ! j ! k !)^{2}} \\
& \leq\left.\frac{\gamma^{n}}{n !} \sum_{\substack{0 \leq i, j, k \leq n \\
i+j+k=n}} \frac{n !}{i ! j ! k !}\right]^{2} \cdot
\end{aligned}
$$

It is known that

$$
\sum \frac{n !}{i ! j ! k !}=3^{n}
$$

This immediately implies that

$$
\frac{\gamma^{n}}{n !}\left[\sum_{\substack{0 \leq i, j, k \leq n \\ i+j+k=n}} \frac{n !}{i ! j ! k !}\right]^{2}=\frac{\gamma^{n}}{n !} 3^{(2 n)}=\frac{(9 \gamma)^{n}}{n !} .
$$

Together with estimates (31) and (32), the latter relation yields the a priori estimate

$$
\left|\left(\left(A_{1}+A_{2}+A_{3}\right)^{n} v\right)(x, y)\right| \leq \frac{(9 \gamma)^{n}}{n !} \text { in } D,
$$

hence the estimate

$$
\left\|\left(A_{1}+A_{2}+A_{3}\right)^{n}\right\|=\max _{\substack{v \in S \\(x, y) \in D}}\left|\left(\left(A_{1}+A_{2}+A_{3}\right)^{n} v\right)(x, y)\right| \leq \frac{(9 \gamma)^{n}}{n !} .
$$

This implies the required inequality, together with the estimate

$$
\left\|(I+A)^{-1}\right\| \leq\left\|\sum_{n=0}^{\infty} A^{n}\right\| \leq \sum_{n=0}^{\infty}\left\|A^{n}\right\| \leq e^{9 \gamma} .
$$


Now we pass to estimating the operator $B$. Applying to the operator $B$ standard estimates for integral operators in spaces of continuous functions, we obtain

$$
\begin{aligned}
\|B\|=\max _{\substack{v \in S \\
(x, y) \in D}} \mid & -\frac{a(x, y)}{\kappa_{2}} \int_{0}^{Y} K_{2}(\eta) \int_{0}^{\eta} v(x, t) d t d \eta \\
& -\frac{b(x, y)}{\kappa_{1}} \int_{0}^{X} K_{1}(\xi) \int_{0}^{\xi} v(s, y) d s d \xi \\
& -\frac{c(x, y)}{\kappa_{1}} \int_{0}^{X} K_{1}(\xi) \int_{0}^{\xi} \int_{0}^{y} v(s, t) d t d s d \xi \\
& \quad-\frac{c(x, y)}{\kappa_{1}} \int_{0}^{Y} K_{2}(\eta) \int_{0}^{x} \int_{0}^{\eta} v(s, t) d t d s d \eta \\
& +\frac{c(x, y)}{\kappa_{1} \kappa_{2}} \int_{0}^{X} \int_{0}^{Y} K_{1}(\xi) K_{2}(\eta) \int_{0}^{\xi} \int_{0}^{\eta} v(s, t) d t d s d \eta d \xi \mid \\
\leq & \frac{|a|}{\left|\kappa_{2}\right|} \int_{0}^{Y} \eta\left|K_{2}(\eta)\right| d \eta+\frac{|b|}{\left|\kappa_{1}\right|} \int_{0}^{X} \xi\left|K_{1}(\xi)\right| d \xi \\
& +\frac{|c|}{\left|\kappa_{1}\right|} y \int_{0}^{X} \xi\left|K_{1}(\xi)\right| d \xi+\frac{|c|}{\left|\kappa_{2}\right|} x \int_{0}^{Y} \eta\left|K_{2}(\eta)\right| d \eta \\
& +\frac{|c|}{\left|\kappa_{1} \kappa_{2}\right|} \int_{0}^{X} \xi\left|K_{1}(\xi)\right| d \xi \int_{0}^{Y} \eta\left|K_{1}(\eta)\right| d \eta \\
= & |a| Y \bar{\kappa}_{2}+|b| X \bar{\kappa}_{1}+|c| y X \bar{k}_{1}+|c| x Y \bar{k}_{2}+|c| X Y \bar{k}_{1} \bar{k}_{2} \\
\leq & (|a| Y+|b| X+3|c| X Y) \bar{\kappa} \leq 5 \gamma \bar{\kappa} .
\end{aligned}
$$

Combining the obtained estimates for the operators $(I+A)^{-1}$ and $B$ with condition (17), we arrive at the required result (201):

$$
\left\|(I+A)^{-1} B\right\| \leq\left\|(I+A)^{-1}\right\|\|B\| \leq 5 \gamma \bar{\kappa} e^{9 \gamma}<1 .
$$

Remark 2. It is important to note that if the functions $K_{1}$ and $K_{2}$ do not change sign on their range intervals, then inequality (17) always holds in a sufficiently small region. In other words, problem $(\mathcal{P})$ is locally solvable regardless of parameters that determine the operator of the problem.

Proof. If the functions $K_{1}(x)$ and $K_{2}(y)$ do not change sign on $I_{x}$ and $I_{y}$, respectively, we have

$$
\begin{aligned}
& \bar{\kappa}_{1}=\frac{1}{X\left|\kappa_{1}\right|} \int_{0}^{X} \xi\left|K_{1}(\xi)\right| d \xi \leq \frac{1}{X\left|\kappa_{1}\right|} X \int_{0}^{X}\left|K_{1}(\xi)\right| d \xi=1, \\
& \bar{\kappa}_{2}=\frac{1}{Y\left|\kappa_{2}\right|} \int_{0}^{Y} \eta\left|K_{2}(\eta)\right| d \eta \leq \frac{1}{Y\left|\kappa_{2}\right|} Y \int_{0}^{Y}\left|K_{2}(\eta)\right| d \eta=1 .
\end{aligned}
$$

Hence also $\bar{\kappa} \leq 1$. On the other hand, the function $e^{-9 \gamma} / 5 \gamma$ tends to infinity as $\gamma \rightarrow 0$. Therefore, inequality (17) holds for sufficiently small $\gamma$. By the definition of the constant $\gamma$ the convergence of $\gamma$ to zero is equivalent to convergence of the diameter of the region $D$. This means that problem $(\mathcal{P})$ is locally solvable. 
Remark 3. The obtained result-unique solvability of problem $(\mathcal{P})$ in each sufficiently small region - turns out to be sharp, as is illustrated by the following example:

$$
\left\{\begin{aligned}
u_{x y}-u=0, & \text { in int } D \\
\int_{0}^{2 \pi} \sin x e^{-x} u(x, y) d x=0, & y \in I_{y} \\
\int_{0}^{2 \pi} \sin y e^{-y} u(x, y) d y=0, & x \in I_{x} .
\end{aligned}\right.
$$

One can easily see that

$$
u(x, y)=c e^{x+y}
$$

is a solution of this problem for each real value of $c$. So, in this case, the solution is not unique.

1.5. The Fredholm property of problem $(\mathcal{P})$. Remark 3 shows that for some choices of functions $a(x, y), b(x, y), c(x, y), K_{1}(x), K_{2}(y)$ and of the region $D$, problem $(\mathcal{P})$ is not uniquely solvable. In this subsection we study conditions implying that the operator corresponding to this problem is Fredholm. The next theorem states a sufficient condition for problem $(\mathcal{P})$ to be Fredholm. Remark 5 below shows that this condition is sharp: if it is violated, the problem may become non-Fredholm.

Theorem 1.5. Let us assume that in problem $(\mathcal{P})$ we have $b(x, y)=b(x)$. Let

and

$$
\widetilde{K}_{1}(x):=K_{1}(x) \exp \left\{-\int_{0}^{x} b(s) d s\right\}
$$

Under the conditions

$$
\widetilde{\kappa}_{1}=\int_{0}^{X} \widetilde{K}_{1}(x) d x
$$

$$
\widetilde{\kappa}_{1} \kappa_{2} \neq 0 \quad \text { and } \quad \int_{0}^{Y} K_{2}(y) \exp \left\{-\int_{0}^{y} a(x, t) d t\right\} d y \neq 0, \quad \text { for all } \quad x \in I_{x},
$$

problem $(\mathcal{P})$ is Fredholm.

Proof. Introduce the functions

$$
\begin{gathered}
\widetilde{c}(x, y):=c(x, y)-a(x, y) b(x), \\
\widetilde{f}(x, y):=f(x, y) \exp \left\{\int_{0}^{x} b(s) d s\right\}, \quad \widetilde{\varphi}(x):=\varphi(x) \exp \left\{\int_{0}^{x} b(s) d s\right\} .
\end{gathered}
$$

Replacing $u(x, y)$ in $(\mathcal{P})$ with the new unknown function

$$
w(x, y)=u(x, y) \exp \left\{\int_{0}^{x} b(s) d s\right\},
$$

we arrive at the equivalent problem

$$
\left\{\begin{array}{l}
w_{x y}+a w_{x}+\widetilde{c} w=\widetilde{f} \text { in int } D, \\
\int_{0}^{X} \widetilde{K}_{1}(x) w(x, y) d x=\psi(y), \quad y \in I_{y}, \\
\int_{0}^{Y} K_{2}(y) w(x, y) d y=\widetilde{\varphi}(x), \quad x \in I_{x} .
\end{array}\right.
$$

Let us note that compatibility condition (3) takes the form

$$
\int_{0}^{X} \widetilde{K}_{1}(x) \widetilde{\varphi}(x) d x=\int_{0}^{Y} K_{2}(y) \psi(y) d y .
$$


To prove that problem (34) is Fredholm, we use the following approach. First we establish that the integral operator $\mathcal{C}$ corresponding to the operator generated by the left-hand side of relations (34) is Fredholm; for simplicity, we will denote this operator by $\mathcal{P}$ as well. Then we prove that $\operatorname{dim} \operatorname{ker} \mathcal{P}$ and $\operatorname{dim} \operatorname{coker} \mathcal{P}$ are bounded from above by the number $\operatorname{dim} \operatorname{ker} \mathcal{C}=\operatorname{dim} \operatorname{coker} \mathcal{C}$. Finally, we establish that the range of the operator $\mathcal{P}$ is closed.

In our case, the integral equation (13) corresponding to problem $(\mathcal{P})$ takes the form

$$
\begin{aligned}
& \mathcal{C} w:= w(x, y)+a(x, y)\left[\int_{0}^{y} w(x, t) d t-\frac{1}{\kappa_{2}} \int_{0}^{Y} K_{2}(\eta) \int_{0}^{\eta} w(x, t) d t d \eta\right] \\
&+\widetilde{c}(x, y)\left[\int_{0}^{x} \int_{0}^{y} w(s, t) d t d s-\frac{1}{\widetilde{\kappa}_{1}} \int_{0}^{X} \widetilde{K}_{1}(\xi) \int_{0}^{\xi} \int_{0}^{y} w(s, t) d t d s d \xi\right. \\
&-\frac{1}{\kappa_{2}} \int_{0}^{Y} K_{2}(\eta) \int_{0}^{x} \int_{0}^{\eta} w(s, t) d t d s d \eta \\
&\left.+\frac{1}{\widetilde{\kappa}_{1} \kappa_{2}} \int_{0}^{X} \int_{0}^{Y} \widetilde{K}_{1}(\xi) K_{2}(\eta) \int_{0}^{\xi} \int_{0}^{\eta} w(s, t) d t d s d \eta d \xi\right] \\
&= \widetilde{f}(x, y)-\frac{a(x, y)}{\kappa_{2}} \widetilde{\varphi}^{\prime}(x) \\
&+\widetilde{c}(x, y)\left(\frac{1}{\widetilde{\kappa}_{1} \kappa_{2}} \int_{0}^{Y} K_{2}(\eta) \psi(\eta) d \eta-\frac{\widetilde{\varphi}(x)}{\kappa_{2}}-\frac{\psi(y)}{\widetilde{\kappa}_{1}}\right) .
\end{aligned}
$$

Let us introduce the following integral operators in the space $C(D)$ :

$$
\begin{aligned}
& \left(A_{1} w\right)(x, y):=a(x, y) \int_{0}^{y} w(x, t) d t \text { in } D, \\
& \left(A_{2} w\right)(x, y):=-\frac{a(x, y)}{\kappa_{2}} \int_{0}^{Y} K_{2}(\eta) \int_{0}^{\eta} w(x, t) d t d \eta \text { in } D
\end{aligned}
$$

and

$$
\begin{aligned}
(B w)(x, y):=\widetilde{c}(x, y)( & \int_{0}^{x} \int_{0}^{y} w(s, t) d t d s \\
& -\frac{1}{\widetilde{\kappa}_{1}} \int_{0}^{X} \widetilde{K}_{1}(\xi) \int_{0}^{\xi} \int_{0}^{y} w(s, t) d t d s d \xi \\
& -\frac{1}{\kappa_{2}} \int_{0}^{Y} K_{2}(\eta) \int_{0}^{x} \int_{0}^{\eta} w(s, t) d t d s d \eta \\
& \left.+\frac{1}{\widetilde{\kappa}_{1} \kappa_{2}} \int_{0}^{X} \int_{0}^{Y} \widetilde{K}_{1}(\xi) K_{2}(\eta) \int_{0}^{\xi} \int_{0}^{\eta} w(s, t) d t d s d \eta d \xi\right) \text { in } D .
\end{aligned}
$$

Also let

$$
\begin{aligned}
\widetilde{f}_{1}(x, y):= & \widetilde{f}(x, y)-\frac{a(x, y)}{\kappa_{2}} \widetilde{\varphi}^{\prime}(x) \\
& +\widetilde{c}(x, y)\left(\frac{1}{\widetilde{\kappa}_{1} \kappa_{2}} \int_{0}^{Y} K_{2}(\eta) \psi(\eta) d \eta-\frac{\widetilde{\varphi}(x)}{\kappa_{2}}-\frac{\psi(y)}{\widetilde{\kappa}_{1}}\right) .
\end{aligned}
$$

In the operator form, the integral equation (35) looks as follows:

$$
\mathcal{C} w:=\left(I+A_{1}+A_{2}\right) w+B w=\tilde{f}_{1} .
$$


Our next goal is to prove that the operator $\mathcal{C}$ is Fredholm. By an easy generalization of the Riesz-Schauder theorem, it suffices to prove that the operator $I+A_{1}+A_{2}$ is invertible and the operator $B$ is compact.

Lemma 1.6. Let $p=p(x, y)$ and $q=q(x)$ be arbitrary continuous functions in $D$ and on $I_{x}$, respectively. Then for each integer $n \geq 1$ we have

$$
\left(A_{1}^{n}(p q)\right)(x, y)=q(x)\left(A_{1}^{n} p\right)(x, y) \quad \text { in } D .
$$

Proof. For $n=1$ the result is clear:

$$
\begin{aligned}
\left(A_{1}(p q)\right)(x, y) & =a(x, y) \int_{0}^{y} p(x, t) q(x) d t \\
& =q(x) a(x, y) \int_{0}^{y} p(x, t) d t=q(x)\left(A_{1} p\right)(x, y) .
\end{aligned}
$$

If it is true for some $k \geq 1$, i.e.,

$$
\left(A_{1}^{k}(p q)\right)(x, y)=q(x)\left(A_{1}^{k} p\right)(x, y) \text { in } D,
$$

then

$$
\begin{aligned}
\left(A_{1}^{k+1}(p q)\right)(x, y) & =a(x, y) \int_{0}^{y}\left(A_{1}^{k}(p q)\right)(x, t) d t \\
& =a(x, y) q(x) \int_{0}^{y}\left(A_{1}^{k}(p)\right)(x, t) d t=q(x)\left(A_{1}^{k+1}(p)\right)(x, y),
\end{aligned}
$$

and by the induction hypothesis the required relation is proved.

Lemma 1.7. We have

$$
\sum_{n=0}^{\infty}(-1)^{n}\left(A_{1}^{n} a\right)(x, y)=a(x, y) \exp \left\{-\int_{0}^{y} a(x, t) d t\right\} \quad \text { in } D .
$$

Proof. Introduce the notation

$$
\omega(x, y):=\int_{0}^{y} a(x, t) d t
$$

and prove that for each integer $n \geq 0$ we have

$$
\left(A_{1}^{n} a\right)(x, y)=a(x, y) \frac{\omega^{n}(x, y)}{n !} .
$$

For $n=0$ and $n=1$ the result is clear. Let us assume that it holds for $n=k \geq 1$ and prove that it holds for $n=k+1$ as well:

$$
\begin{aligned}
\left(A_{1}^{k+1} a\right)(x, y) & =\frac{\left(A_{1}\left(a \omega^{k}\right)\right)(x, y)}{k !}=\frac{a(x, y)}{k !} \int_{0}^{y} a(x, t) \omega^{k}(x, t) d t \\
& =\frac{a(x, y)}{k !} \int_{0}^{y}\left[\frac{\partial}{\partial t} \omega(x, t)\right] \omega^{k}(x, t) d t=\frac{a(x, y)}{(k+1) !} \int_{0}^{y} \frac{\partial}{\partial t}\left[\omega^{k+1}(x, t)\right] d t \\
& =\frac{a(x, y)}{(k+1) !}\left(\omega^{k+1}(x, y)-\omega^{k+1}(x, 0)\right)=\frac{a(x, y) \omega^{k+1}(x, y)}{(k+1) !} .
\end{aligned}
$$

Thus the required relation is proved, from which it follows immediately that

Since

$$
\left|\left(A_{1}^{n} a\right)(x, y)\right|=\frac{1}{n !}\left|a(x, y)\left(\int_{0}^{y} a(x, t) d t\right)^{n}\right| \leq \frac{|a|(Y|a|)^{n}}{n !} .
$$

$$
\sum_{n=0}^{\infty} \frac{|a|(Y|a|)^{n}}{n !}=|a| \cdot \exp \{Y|a|\}
$$


we see that the series $\sum_{n=0}^{\infty}(-1)^{n}\left(A_{1}^{n} u\right)(x, y)$ uniformly converges, and its sum equals

$$
a(x, y) \exp \left\{-\int_{0}^{y} a(x, t) d t\right\} .
$$

Lemma 1.8. Under the condition

$$
\int_{0}^{Y} K_{2}(y) \exp \left\{-\int_{0}^{y} a(x, t) d t\right\} d y \neq 0 \quad \text { for all } x \in I_{x},
$$

the operator $I+A_{1}+A_{2}$ is invertible in the space $C(D)$.

Proof. We prove that for each function $f \in C(D)$ the equation

$$
\left(I+A_{1}+A_{2}\right) w=f
$$

has a unique solution $w \in C(D)$. First let us note that, according to (24), the operator $A_{1}$ has the property

$$
\sum_{n=0}^{\infty}\left\|A_{1}^{n}\right\| \leq \exp \{|a| Y\}
$$

Indeed, it suffices to recall that, according to (24),

$$
\left\|A_{1}^{n}\right\|=\max _{\substack{(x, y) \in D \\ v \in S}}\left|A_{1} v(x, y)\right| \leq \frac{(|a| Y)^{n}}{n !} .
$$

Therefore the operator $I+A_{1}$ is invertible and

$$
\left(I+A_{1}\right)^{-1}=\sum_{n=0}^{\infty}(-1)^{n} A_{1}^{n}
$$

Introduce the notation

$$
(K w)(x)=\int_{0}^{Y} K_{2}(y) \int_{0}^{y} w(x, t) d t d y .
$$

Then (38) can be rewritten in the following equivalent form:

or

$$
\left\{\left[I+\left(I+A_{1}\right)^{-1}\left(-\frac{a}{\kappa_{2}} K\right)\right] w\right\}(x, y)=\left[\left(I+A_{1}\right)^{-1} f\right](x, y)
$$

$$
w(x, y)+\left(I+A_{1}\right)^{-1}\left(-\frac{a}{\kappa_{2}}(K w)\right)(x, y)=\left[\left(I+A_{1}\right)^{-1} f\right](x, y) .
$$

According to (39) we have

$$
\left[w+\sum_{n=0}^{\infty}(-1)^{n} A_{1}^{n}\left(-\frac{a}{\kappa_{2}}(K w)\right)\right](x, y)=\left[\left(I+A_{1}\right)^{-1} f\right](x, y) .
$$

Since the function $K w$ does not depend on $y$, Lemma 1.6 shows that this relation is equivalent to

$$
\left[w-\frac{1}{\kappa_{2}}\left(\sum_{n=0}^{\infty}(-1)^{n}\left(A_{1}^{n} a\right)\right)(K w)\right](x, y)=\left[\left(I+A_{1}\right)^{-1} f\right](x, y) .
$$

Introduce the notation

$$
\begin{aligned}
\widetilde{a}(x, y) & :=-\frac{1}{\kappa_{2}} a(x, y) \exp \left\{-\int_{0}^{y} a(x, t) d t\right\}, \\
f_{1}(x, y) & :=\left(I+A_{1}\right)^{-1} f .
\end{aligned}
$$


According to Lemma 1.7, the function $w(x, y)$ is a solution of the integral equation

$$
w(x, y)+\widetilde{a}(x, y) \int_{0}^{Y} K_{2}(\eta) \int_{0}^{\eta} w(x, t) d t d \eta=f_{1}(x, y)
$$

and hence satisfies the relation

$$
\begin{aligned}
\int_{0}^{Y} & K_{2}(y) \int_{0}^{y}\left(w(x, t)+\widetilde{a}(x, t) \int_{0}^{Y} K_{2}(z) \int_{0}^{z} w(x, s) d s d z\right) d t d y \\
& =\int_{0}^{Y} K_{2}(y) \int_{0}^{y} f_{1}(x, t) d t d y .
\end{aligned}
$$

Together with (40) this yields

$$
\begin{aligned}
& \left(\int_{0}^{Y} K_{2}(y) \int_{0}^{y} w(x, t) d t d y\right)\left(1+\int_{0}^{Y} K_{2}(y) \int_{0}^{y} \widetilde{a}(x, t) d t d y\right) \\
& \quad=\int_{0}^{Y} K_{2}(y) \int_{0}^{y} f_{1}(x, t) d t d y .
\end{aligned}
$$

Moreover,

$$
\begin{aligned}
\int_{0}^{Y} K_{2}(y) \int_{0}^{y} \widetilde{a}(x, t) d t d y & =-\frac{1}{\kappa_{2}} \int_{0}^{Y} K_{2}(y)\left(\int_{0}^{y} a(x, t) \exp \left\{-\int_{0}^{t} a(x, \bar{t}) d \bar{t}\right\} d t\right) d y \\
& =\frac{1}{\kappa_{2}} \int_{0}^{Y} K_{2}(y)\left(\int_{0}^{y} \frac{\partial}{\partial t} \exp \left\{-\int_{0}^{t} a(x, \bar{t}) d \bar{t}\right\} d t\right) d y \\
& =\frac{1}{\kappa_{2}} \int_{0}^{Y} K_{2}(y)\left(\exp \left\{-\int_{0}^{y} a(x, t) d t\right\}-1\right) d y \\
& =-1+\frac{1}{\kappa_{2}} \int_{0}^{Y} K_{2}(y) \exp \left\{-\int_{0}^{y} a(x, t) d t\right\} d y .
\end{aligned}
$$

Combining the two latter relations and using (37) we find that

$$
\begin{aligned}
& \int_{0}^{Y} K_{2}(y) \int_{0}^{y} w(x, t) d t d y \\
& \quad=\frac{\kappa_{2}}{\int_{0}^{Y} K_{2}(y) \exp \left\{-\int_{0}^{y} a(x, t) d t\right\} d y} \int_{0}^{Y} K_{2}(y) \int_{0}^{y} f_{1}(x, t) d t d y .
\end{aligned}
$$

Substituting the right-hand side of (43) in (41), we find the unique solution of the integral equation (38) in the form

$$
w(x, y)=f_{1}(x, y)-\frac{\kappa_{2} \widetilde{a}(x, y)}{\int_{0}^{Y} K_{2}(y) \exp \left\{-\int_{0}^{y} a(x, t) d t\right\} d y} \int_{0}^{Y} K_{2}(y) \int_{0}^{y} f_{1}(x, t) d t d y .
$$

Lemma 1.9. The operator $B$ defined in (36) is compact in the space $C(D)$. 
Proof. Introduce the notation

$$
\begin{aligned}
& \left(B_{1} w\right)(x, y):=\widetilde{c}(x, y) \int_{0}^{x} \int_{0}^{y} w(s, t) d t d s \text { in } D \\
& \left(B_{2} w\right)(x, y):=-\frac{\widetilde{c}(x, y)}{\widetilde{\kappa}_{1}} \int_{0}^{X} \widetilde{K}_{1}(\xi) \int_{0}^{\xi} \int_{0}^{y} w(s, t) d t d s d \xi \quad \text { in } D, \\
& \left(B_{3} w\right)(x, y):=-\frac{\widetilde{c}(x, y)}{\kappa_{2}} \int_{0}^{Y} K_{2}(\eta) \int_{0}^{x} \int_{0}^{\eta} w(s, t) d t d s d \eta \quad \text { in } D, \\
& \left(B_{4} w\right)(x, y)=: \frac{\widetilde{c}(x, y)}{\widetilde{\kappa}_{1} \kappa_{2}} \int_{0}^{X} \int_{0}^{Y} \widetilde{K}_{1}(\xi) K_{2}(\eta) \int_{0}^{\xi} \int_{0}^{\eta} w(s, t) d t d s d \eta d \xi \quad \text { in } D .
\end{aligned}
$$

Then

$$
(B w)(x, y)=\left[\left(B_{1}+B_{2}+B_{3}+B_{4}\right) w\right](x, y) .
$$

Let us verify that each operator $B_{j}$ is compact. Take an arbitrary bounded set $M \subset C(D)$ and prove that for each $j=1,2,3,4$ its image $B_{j}(M)$ is precompact, i.e., the closure $\overline{\mathcal{B}_{j}(M)}$ is compact. Introduce the operator

$$
\left(\widetilde{B}_{1} w\right)(x, y):=\int_{0}^{x} \int_{0}^{y} w(s, t) d t d s \quad \text { in } D
$$

and denote by $Q_{1}$ the closure of the image $\widetilde{\mathcal{B}}_{1}(M)$ of the set $M$ under the operator $\widetilde{\mathcal{B}_{1}}$. Let

$$
|w| \leq m
$$

for all $w \in M$. Then for all these $w$ 's we have

$$
\left|\widetilde{\mathcal{B}}_{1} w\right|=\left|\int_{0}^{X} \int_{0}^{Y} w(s, t) d s d t\right| \leq X Y m
$$

implying that the set $Q_{1}$ is uniformly bounded. To prove that it is equicontinuous (which guarantees the compactness of $Q_{1}$ according to the Arcela theorem) we choose an arbitrary $\varepsilon>0$. For a point $(x, y) \in D$, by $B_{(x, y)}^{r}$ we denote the ball of radius $r$ centered at $(x, y)$. Then for an arbitrary function $v \in Q_{1}$ and each point $\left(x_{1}, y_{1}\right) \in D \cap B_{(x, y)}^{r}$ we have

$$
\begin{gathered}
\left|v\left(x_{1}, y_{1}\right)-v(x, y)\right|=\left|\int_{0}^{x_{1}} \int_{0}^{y_{1}} w(s, t) d t d s-\int_{0}^{x} \int_{0}^{y} w(s, t) d t d s\right| \\
=\mid \int_{0}^{x_{1}} \int_{0}^{y_{1}} w(s, t) d t d s-\int_{0}^{x_{1}} \int_{0}^{y} w(s, t) d t d s \\
\quad+\int_{0}^{x_{1}} \int_{0}^{y} w(s, t) d t d s-\int_{0}^{x} \int_{0}^{y} w(s, t) d t d s \mid \\
\leq\left|\int_{0}^{x_{1}} \int_{y_{1}}^{y} w(s, t) d t d s\right|+\left|\int_{x}^{x_{1}} \int_{0}^{y} w(s, t) d t d s\right| \\
\leq M\left(X\left|y_{1}-y\right|+Y\left|x_{1}-x\right|\right) \leq M r(X+Y) .
\end{gathered}
$$

It is clear that if

$$
r \leq \frac{\varepsilon}{(X+Y) M}
$$

then the left-hand side of (44) does not exceed $\varepsilon$. Therefore, for an arbitrary function $v \in Q_{1}$ and for each $\delta<r$ we have

$$
\sup _{\substack{(x, y) \in D \\\left(y_{1}\right) \in D \cap B_{(x, y)}^{\delta}}}\left|v\left(x_{1}, y_{1}\right)-v(x, y)\right| \leq \varepsilon .
$$


The latter inequality means that, as required, the set $\widetilde{\mathcal{B}}_{1}(M)$ is equicontinuous, so that the operator $\widetilde{\mathcal{B}}_{1}$ is compact. Since the multiplication operator

$$
w(x, y) \rightarrow c(x, y) w(x, y)
$$

is bounded in the space $C(D)$, its composition with the compact operator $\widetilde{B}_{1}$ is also compact.

Now we verify that the operator

$$
\widetilde{B}_{2}: w \rightarrow \int_{0}^{X} \widetilde{K}_{1}(\xi) \int_{0}^{\xi} \int_{0}^{y} w(s, t) d t d s d \xi \quad \text { in } D
$$

is compact. Let $M$ and $Q_{2}$ have the same meaning as $M$ and $Q_{1}$ for the operator $\widetilde{\mathcal{B}}_{1}$. We prove that the set $Q_{2}$ is compact in $C(D)$. Since $Q_{2}$ is bounded due to the estimate

$$
\left|\widetilde{B}_{2} w(y)\right|=\left|\int_{0}^{X} \widetilde{K}_{1}(\xi) \int_{0}^{\xi} \int_{0}^{y} w(s, t) d t d s d \xi\right| \leq m X Y \int_{0}^{X}\left|\widetilde{K}_{1}(\xi)\right| d \xi
$$

for each function $w \in M$, it remains to prove that the set $Q_{2}$ is equicontinuous. Choose an arbitrary $\varepsilon>0$ and two arbitrary points $y$ and $y_{1}$ on the interval $I_{y}$ such that

$$
\left|y-y_{1}\right|<r
$$

where $r$ will be fixed later. Then for each function $v \in Q_{2}$ we have

$$
\begin{aligned}
& \left|v\left(y_{1}\right)-v(y)\right| \\
& \quad=\left|\int_{0}^{X} \widetilde{K}_{1}(\xi) \int_{0}^{\xi} \int_{0}^{y_{1}} w(s, t) d t d s d \xi-\int_{0}^{X} \widetilde{K}_{1}(\xi) \int_{0}^{\xi} \int_{0}^{y} w(s, t) d t d s d \xi\right| \\
& \quad \leq m X\left|y_{1}-y\right| \int_{0}^{X}\left|\widetilde{K}_{1}(\xi)\right| d \xi \leq m X r \int_{0}^{X}\left|\widetilde{K}_{1}(\xi)\right| d \xi .
\end{aligned}
$$

It follows that if

$$
r<\frac{\varepsilon}{m X \int_{0}^{X}\left|\widetilde{K}_{1}(\xi)\right| d \xi},
$$

then the left-hand side in (45) does not exceed $\varepsilon$, as required. Finally, as before, the compactness of the operator $\mathcal{B}=c \widetilde{\mathcal{B}}_{2}$ follows from the fact that the subspace of compact operators is invariant under multiplication by a continuous function.

Since the operator $\mathcal{B}_{3}$ has the same form as the operator $\mathcal{B}_{2}$, to complete the proof of Lemma 1.9 it suffices to establish the compactness of the operator

$$
\left(\widetilde{B}_{4} w\right)(x, y):=\int_{0}^{X} \int_{0}^{Y} \widetilde{K}_{1}(\xi) K_{2}(\eta) \int_{0}^{\xi} \int_{0}^{\eta} w(s, t) d t d s d \eta d \xi \quad \text { in } D .
$$

This immediately follows from the boundedness of this operator and from the fact that its image is one-dimensional.

Summarizing, we can say that since, as we have proved, the operator $I+A_{1}+A_{2}$ is invertible and the operator $\mathcal{B}$ is compact in the space $C(D)$, the integral operator $\mathcal{C}=I+A_{1}+A_{2}+\mathcal{B}$ is Fredholm.

Now we return to the proof of Theorem 1.5. It remains to prove that

$$
\operatorname{dim} \operatorname{ker} \mathcal{P}<\infty, \quad \operatorname{dim} \operatorname{coker} \mathcal{P}<\infty
$$

and that the range of the operator $\mathcal{P}$ is closed. To each function $u(x, y) \in \operatorname{ker} \mathcal{P}$ we associate the function $v(x, y)=\partial_{x} \partial_{y} u(x, y)$, which, as we have seen, belongs to the subspace $\operatorname{ker} \mathcal{C}$. Let us show that such a map sends linearly independent elements of $\operatorname{ker} \mathcal{P}$ to linearly independent functions. Indeed, let

$$
\left\{u_{j}(x, y)\right\}_{1}^{N} \subset \operatorname{ker} \mathcal{P}
$$


be such that if $\sum_{j=1}^{N} \lambda_{j} u_{j}(x, y)=0$ in $D$, then all numbers $\lambda_{j}$ are equal to 0 . Let us consider $N$ functions

$$
v_{j}(x, y)=\partial_{x} \partial_{y} u_{j}(x, y), \quad j=1, \ldots, N
$$

and assume that they are linearly dependent, so that for some nonzero $N$-tuple $c_{1}, \ldots, c_{N}$ of real numbers we have

$$
c_{1} v_{1}(x, y)+\cdots+c_{N} v_{N}(x, y) \equiv 0, \quad(x, y) \in D .
$$

Consider the function

$$
U(x, y)=\sum_{j=1}^{N} c_{j} u_{j}(x, y) .
$$

From the choice of functions $u_{j}$ it is clear that

$$
\partial_{x} \partial_{y} U(x, y)=0, \quad\left(K_{1} u\right)(x)=0, \quad\left(K_{2} u\right)(y)=0
$$

at all points $(x, y) \in D$. But according to Proposition 1.1 this means that $U(x, y) \equiv 0$, contradicting the linear independence of the functions $u_{j}(x, y)$. Hence we have proved that

Now we prove that

$$
\operatorname{dim} \operatorname{ker} \mathcal{P} \leq \operatorname{dim} \operatorname{ker} \mathcal{C}
$$

Indeed, choose 3-vectors

$$
\operatorname{dim} \text { coker } \mathcal{P} \leq \operatorname{dim} \text { coker } \mathcal{C}
$$

$$
Z_{j}=\left\{f_{j}(x, y), \varphi_{j}(x), \psi_{j}(y)\right\}_{1}^{N}
$$

that are not in the domain $\mathcal{R}(\mathcal{P})$ of the operator $\mathcal{P}$. By Theorem 1.2 the corresponding functions

$$
\mathcal{H}_{j}(x, y)=f_{j}(x, y)-\ell\left(\varphi_{j}(x), \psi_{j}(y)\right)
$$

where

$$
\begin{aligned}
\ell(\varphi(x), \psi(y)):= & \frac{a(x, y)}{\kappa_{2}} \varphi^{\prime}(x)+\frac{b(x, y)}{\kappa_{1}} \psi^{\prime}(y) \\
& +\frac{c(x, y)}{\kappa_{1} \kappa_{2}}\left(\frac{1}{2} \int_{0}^{X} K_{1}(x) \varphi(x) d x\right. \\
& \left.+\frac{1}{2} \int_{0}^{Y} K_{2}(y) \psi(y) d y-\kappa_{1} \varphi(x)-\kappa_{2} \psi(y)\right)
\end{aligned}
$$

do not belong to the domain of the operator $\mathcal{C}$. As before, if the selected 3-vectors $Z_{j}$ are linearly independent modulo the space $\mathcal{R}(\mathcal{P})$, then the same Theorem 1.2 shows that the above functions $\mathcal{H}_{j}(x, y)$ are linearly independent modulo the space $\mathcal{R}(\mathcal{C})$. This immediately leads to inequality (47), hence inequalities (46).

To prove that the domain of the operator $\mathcal{P}$ is closed, consider a sequence of elements

$$
\left(f_{n}, \varphi_{n}, \psi_{n}\right)=\mathcal{P} u_{n}, \quad u_{n} \in \mathcal{W},
$$

in the space $\mathcal{V}$ converging to $(f, \varphi, \psi)$ as $n \rightarrow \infty$. Since the operator $\ell$ is continuous, the sequence of functions

$$
F_{n}=f_{n}-\ell\left(\varphi_{n}, \psi_{n}\right)
$$

converges in the space $C(D)$ to some function $F(x, y)$. According to Theorem 1.2, the functions $F_{n}$ can be represented in the form $F_{n}=\mathcal{C} w_{n}$. Since the operator $\mathcal{C}$ is Fredholm, the function $F$ is of the form $F=\mathcal{C} w$ for some $w$. By the same Theorem 1.2 the problem $\mathcal{P} u=(f, \varphi, \psi)$ with $f-\ell(\varphi, \psi)=F$ is solvable, thus proving the required result.

This completes the proof of Theorem 1.5 
Remark 4 . One can easily see that the condition $b(x, y)=b(x)$ in Theorem 1.5 can be replaced by the condition that

$$
\max _{y} \max _{x}|b(x, y)-b(x, 0)| X
$$

is sufficiently small.

Remark 5. The statement of Theorem 1.5] is sharp: if condition (33) fails to hold, problem $(\mathcal{P})$ may lose the Fredholm property.

Indeed, consider the following problem:

$$
\left\{\begin{array}{l}
u_{x y}+\frac{\cos y}{\sin y+2} u_{x}=0 \quad \text { in int } D \\
\int_{0}^{1} u(x, y) d x=0 \text { in } I_{y}, \\
\int_{0}^{2 \pi} \sin y(\sin y+2) u(x, y) d y=0 \text { in } I_{x} .
\end{array}\right.
$$

Condition (33) is violated because one can immediately verify that

$$
\int_{0}^{Y} K_{2}(y) \exp \left\{-\int_{0}^{y} a(x, t) d t\right\} d y=0
$$

for each $x$. For an arbitrary function $f(x) \in C^{1}\left(I_{x}\right)$ the function

$$
u(x, y)=\frac{f(x)-\int_{0}^{1} f(x) d x}{\sin y+2}
$$

is a solution of this problem. But this means that

$$
\operatorname{dim} \operatorname{ker} \mathcal{P}=\infty
$$

so that the problem is not Fredholm.

\section{Nonlocal quasiboundary PRoblem}

In this section we consider the nonlocal problem for the operator $\mathcal{L}$ in the same domain $D$ as in Section 1, but with additional conditions restricting the values of an unknown function not on a two-dimensional subset of $D$ but on a discrete family of intervals of the form $x=x_{j}, 1 \leq j \leq n$, and $y=y_{k}, k=1, \ldots, m$. As before, we will determine conditions that guarantee the unique solvability of this problem, as well as conditions for its being Fredholm. Of course, both problems studied in this paper can be unified if we consider them in the framework of distribution theory. However, this approach does not yield (so far) significantly new results, while requiring certain accuracy and lengthening the exposition.

2.1. Formulation of the problem. In the same domain $D=\{(x, y) \mid 0 \leq x \leq X, 0 \leq$ $y \leq Y\}$ as before, we consider the problem

$$
\left\{\begin{array}{l}
\partial_{x} \partial_{y} u+a(x, y) \partial_{x} u+b(x, y) \partial_{y} u+c(x, y) u=f(x, y), \\
\sum_{j=1}^{n} \alpha_{j} u\left(x_{j}, y\right)=\varphi(y), 0 \leq y \leq Y, \\
\sum_{k=1}^{m} \beta_{k} u\left(x, y_{k}\right)=\psi(x), 0 \leq x \leq X
\end{array}\right.
$$

where $0 \leq x_{j} \leq X, 0 \leq y_{k} \leq Y$ for all values of $j$ and $k$, all $\alpha_{j}$ and $\beta_{k}$ are real numbers, $a(x, y), b(x, y), c(x, y)$, and $f(x, y)$ are continuous functions, and $\varphi(y)$ and $\psi(x)$ are 
continuously differentiable functions. A solution $u(x, y)$ of problem $\left(\mathcal{P}_{d}\right)$ is supposed to be an element of the space $\mathcal{W}$. As in the classical Goursat problem, the functions $\varphi$ and $\psi$ cannot be chosen arbitrarily. Since, by the second and third relations in problem $\left(\mathcal{P}_{d}\right)$ we have

$$
\sum_{k=1}^{m} \sum_{j=1}^{n} \alpha_{j} \beta_{k} u\left(x_{j}, y_{k}\right)=\sum_{k=1}^{m} \beta_{k} \varphi\left(y_{k}\right)
$$

and

the condition

$$
\sum_{j=1}^{n} \sum_{k=1}^{m} \alpha_{j} \beta_{k} u\left(x_{j}, y_{k}\right)=\sum_{j=1}^{n} \alpha_{j} \psi\left(x_{j}\right)
$$

$$
\sum_{j=1}^{n} \alpha_{j} \psi\left(x_{j}\right)=\sum_{k=1}^{m} \beta_{k} \varphi\left(y_{k}\right)
$$

is necessary for problem $\left(\mathcal{P}_{d}\right)$ to be solvable. As we will see later, there are no additional solvability conditions.

Everywhere below, by $\left(\mathcal{P}_{d}\right)$ we denote the linear operator

$$
\mathcal{W} \ni u \rightarrow(f, \varphi, \psi) \in C(D) \times C^{1}\left(I_{x}\right) \times C^{1}\left(I_{y}\right),
$$

defined by the left-hand side of $\left(\mathcal{P}_{d}\right)$.

2.2. A model problem. As before, we will consider problem $\left(\mathcal{P}_{d}\right)$ in the framework of perturbation theory for linear operators. The key role in this consideration is played by the inverse operator corresponding to the following problem:

$$
\begin{aligned}
\partial_{x} \partial_{y} u & =f(x, y) \quad \text { in } D, \\
\sum_{j=1}^{n} \alpha_{j} u\left(x_{j}, y\right) & =\varphi(y) \quad \text { on } I_{x}, \\
\sum_{k=1}^{m} \beta_{k} u\left(x, y_{k}\right) & =\psi(x) \quad \text { on } I_{y} .
\end{aligned}
$$

We assume, of course, that condition (48) is satisfied.

First let $f=0$. Then a generic solution of equation (49) is of the form

$$
u(x, y)=v(x)+w(y),
$$

where $v(x)$ and $w(y)$ are arbitrary functions in the spaces $C^{1}\left(I_{x}\right)$ and $C^{1}\left(I_{y}\right)$, respectively. Conditions (50) and (51) together with (48) yield

$$
\sum_{j=1}^{n} \alpha_{j} v\left(x_{j}\right)+\alpha w(y)=\varphi(y), \quad \sum_{k=1}^{m} \beta_{k} w\left(y_{k}\right)+\beta v(x)=\psi(x),
$$

where

$$
\alpha=\sum_{j=1}^{n} \alpha_{j} \quad \text { and } \quad \beta=\sum_{k=1}^{m} \beta_{k} .
$$

It follows that if $\alpha \beta=0$, then at least one of the functions $\varphi(y), \psi(x)$ should be a constant function, which makes the class of problems under consideration significantly smaller. Therefore, below we assume that condition $\alpha \beta \neq 0$ is satisfied; according to (52), this allows us to represent the function $u(x, y)$ in the form

$$
u(x, y)=\frac{\varphi(y)}{\alpha}+\frac{\psi(x)}{\beta}-\sum_{j=1}^{n} \frac{\alpha_{j}}{\alpha} v\left(x_{j}\right)-\sum_{k=1}^{m} \frac{\beta_{k}}{\beta} w\left(y_{k}\right) .
$$


Since, by (52),

$$
\sum_{j=1}^{n} \frac{\alpha_{j}}{\alpha} v\left(x_{j}\right)+\sum_{k=1}^{m} \frac{\beta_{k}}{\beta} w\left(y_{k}\right)=\sum_{k=1}^{m} \frac{\beta_{k}}{\beta \alpha} \varphi\left(y_{k}\right),
$$

the previous formula yields an explicit solution of problem (49)-(51) with $f=0$ :

$$
u(x, y)=\frac{\varphi(y)}{\alpha}+\frac{\psi(x)}{\beta}-\sum_{k=1}^{m} \frac{\beta_{k} \varphi\left(y_{k}\right)}{\alpha \beta} .
$$

The apparent asymmetry of this solution with respect to the variables $x$ and $y$ is only imaginary: we have to use the necessary condition (48) and rewrite the latter relation in the form

$$
u(x, y)=\frac{\varphi(y)}{\alpha}+\frac{\psi(x)}{\beta}-\frac{1}{2}\left(\sum_{k=1}^{m} \frac{\beta_{k} \varphi\left(y_{k}\right)}{\alpha \beta}+\sum_{j=1}^{n} \frac{\alpha_{j}}{\alpha \beta} \psi\left(x_{j}\right)\right) .
$$

For an arbitrary function $f$, a solution of problem (49)-(51) can be represented as follows:

$$
u(x, y)=F(x, y)+v(x)+w(y),
$$

where

$$
F(x, y)=\int_{0}^{x} \int_{0}^{y} f(s, t) d s d t
$$

and $v(x), w(y)$ are $C^{1}$-functions on the corresponding intervals. Introducing a new unknown function $G=u-F$ we arrive at the problem

$$
\left\{\begin{array}{l}
\partial_{x} \partial_{y} G(x, y)=0 \quad \text { in } \quad D \\
\sum_{j=1}^{n} \alpha_{j} G\left(x_{j}, y\right)=\varphi(y)-\sum_{j=1}^{n} \alpha_{j} F\left(x_{j}, y\right) \\
\sum_{k=1}^{m} \beta_{k} G\left(x, y_{k}\right)=\psi(x)-\sum_{k=1}^{m} \beta_{k} F\left(x, y_{k}\right) .
\end{array}\right.
$$

By previous arguments, the function

$$
\begin{aligned}
G(x, y)= & \frac{\varphi(y)}{\alpha}+\frac{\psi(x)}{\beta}-\sum_{j=1}^{n} \frac{\alpha_{j}}{\alpha} F\left(x_{j}, y\right) \\
& -\sum_{k=1}^{m} \frac{\beta_{k}}{\beta} F\left(x, y_{k}\right)-\sum_{k=1}^{m} \frac{\beta_{k}}{\beta} \varphi\left(y_{k}\right)+\sum_{j=1}^{n} \sum_{k=1}^{m} \frac{\alpha_{j}}{\alpha} \frac{\beta_{k}}{\beta} F\left(x_{j}, y_{k}\right)
\end{aligned}
$$

is a solution of this problem. Therefore, the function

$$
\begin{aligned}
u(x, y)= & F(x, y)-\sum_{j=1}^{n} \frac{\alpha_{j}}{\alpha} F\left(x_{j}, y\right)-\sum_{k=1}^{m} \frac{\beta_{k}}{\beta} F\left(x, y_{k}\right) \\
& -\sum_{j=1}^{n} \sum_{k=1}^{m} \frac{\alpha_{j}}{\alpha} \frac{\beta_{k}}{\beta} F\left(x_{j}, y_{k}\right)+\frac{\varphi(y)}{\alpha}+\frac{\psi(x)}{\beta}-\sum_{k=1}^{m} \frac{\beta_{k} \varphi\left(y_{k}\right)}{\alpha \beta}
\end{aligned}
$$

is the unique solution of the model problem (49)-(51).

If we treat the left-hand side of relations (49)-(51) as a linear operator

$$
\mathcal{P}_{0}: \mathcal{W} \ni u \rightarrow(f, \varphi, \psi) \in C(D) \times C^{1}\left(I_{x}\right) \times C^{1}\left(I_{y}\right),
$$

then the right-hand side of (54) can be interpreted as the inverse operator $\mathcal{P}_{0}^{-1}$, thus proving the existence of this inverse operator. It is the interpretation of our operator $\mathcal{P}_{d}$ as a perturbation of the operator $\mathcal{P}_{0}$ that plays the key role in solving problem $\left(\mathcal{P}_{d}\right)$. 
2.3. Unique solvability of problem $\left(\mathcal{P}_{d}\right)$. In the sequel we use the following notation: for a scalar function $h$ we set $\bar{h}=\max |h|$, and then

$$
\bar{k}_{1}=\frac{\sum_{j=1}^{n}\left|\alpha_{j}\right|}{|\alpha|}, \quad \bar{k}_{2}=\frac{\sum_{j=1}^{m}\left|\beta_{k}\right|}{|\beta|} ; \quad \gamma=\max \{\bar{a} Y, \bar{b} X, \bar{c} X Y\}, \quad \kappa=\max \left\{\bar{k}_{1}, \bar{k}_{2}, \bar{k}_{1} \bar{k}_{2}\right\} .
$$

The main result of this subsection is the following theorem.

Theorem 2.1. If condition (48) is satisfied and

$$
5 \kappa \gamma e^{9 \gamma}<1,
$$

then problem $(\mathcal{P})$ is uniquely solvable in the space $\mathcal{W}$ for all $(f, \varphi, \psi) \in C(D) \times C^{1}\left(I_{x}\right) \times$ $C^{1}\left(I_{y}\right)$.

Proof. Let us represent problem $(\mathcal{P})$ in the operator form

$$
\mathcal{P} u=(f, \varphi, \psi),
$$

where $f \in C(D), \varphi \in C^{1}\left(I_{y}\right), \psi \in C^{1}\left(I_{x}\right)$, and introduce a new unknown function $g \in C(D)$ by the formula

$$
u=\mathcal{P}_{0}^{-1}(g, \varphi, \psi) \text {. }
$$

According to the results of the previous subsection, $\mathcal{P}_{0}$ establishes a one-to-one correspondence between the space $\mathcal{W}$ and the space $C(D) \times C^{1}\left(I_{x}\right) \times C^{1}\left(I_{y}\right)$. Substituting the function $u$ in (56) we arrive at the equation

$$
\left(\mathcal{P} \circ \mathcal{P}_{0}^{-1}\right)(g, \varphi, \psi)=(f, \varphi, \psi),
$$

which is equivalent to problem $(\mathcal{P})$. The role of the unknown function in this equation is played by the function $g$. To rewrite the latter equation explicitly, we use relation (54), where for

$$
F(x, y)=\int_{0}^{x} \int_{0}^{y} g(s, t) d s d t, \quad(x, y) \in I_{x} \times I_{y},
$$

the right-hand side represents the function $\mathcal{P}_{0}^{-1}(g, \varphi, \psi)$. Performing the necessary computations, we arrive at the following form of equation (57), which is the starting point of the proof of Theorem 2.1.

$$
\begin{aligned}
g(x, y) & +a(x, y) \int_{0}^{y} g(x, t) d t+b(x, y) \int_{0}^{x} g(s, y) d s \\
& +c(x, y) \int_{0}^{x} \int_{0}^{y} g(s, t) d s d t-a(x, y) \sum_{k=1}^{m} \frac{\beta_{k}}{\beta} \int_{0}^{y_{k}} g(x, y) d y \\
& -b(x, y) \sum_{j=1}^{n} \frac{\alpha_{j}}{\alpha} \int_{0}^{x_{j}} g(x, y) d x \\
& +c(x, y)\left(\sum_{j=1}^{n} \frac{\alpha_{j}}{\alpha} \int_{0}^{x_{j}} \int_{0}^{y} g(x, t) d x d t+\sum_{k=1}^{m} \frac{\beta_{k}}{\beta} \int_{0}^{y_{k}} \int_{0}^{x} g(s, y) d s d y\right. \\
& \left.\quad+\sum_{j=1}^{n} \sum_{k=1}^{m} \frac{\alpha_{j}}{\alpha} \frac{\beta_{k}}{\beta} \int_{0}^{x_{j}} \int_{0}^{y_{k}} g(x, y) d x d y\right) \\
= & \widetilde{f}(x, y),
\end{aligned}
$$

where

$$
\begin{aligned}
\tilde{f}(x, y)= & f(x, y)-a(x, y) \psi^{\prime}(x)-b(x, y) \varphi^{\prime} \\
& -c(x, y)\left(\sum_{j=1}^{n} \frac{\alpha_{j} \psi\left(x_{j}\right)}{\alpha \beta}-\frac{\varphi(y)}{\alpha}-\frac{\psi(x)}{\beta}\right) .
\end{aligned}
$$


Here both the given function $f(x, y)$ and the unknown function $g(x, y)$ are assumed to be continuous in $D$.

Similarly to the proof of Theorem 1.3 , we introduce the operators

$$
A g:=a(x, y) \int_{0}^{y} g(x, t) d t+b(x, y) \int_{0}^{x} g(s, y) d s+c(x, y) \int_{0}^{x} \int_{0}^{y} g(s, t) d s d t
$$

and

$$
\begin{aligned}
B g:=- & a(x, y) \sum_{k=1}^{m} \frac{\beta_{k}}{\beta} \int_{0}^{y_{k}} g(x, y) d y-b(x, y) \sum_{j=1}^{n} \frac{\alpha_{j}}{\alpha} \int_{0}^{x_{j}} g(x, y) d x \\
& +c(x, y)\left(\sum_{j=1}^{n} \frac{\alpha_{j}}{\alpha} \int_{0}^{x_{j}} \int_{0}^{y} g(x, t) d x d t+\sum_{k=1}^{m} \frac{\beta_{k}}{\beta} \int_{0}^{y_{k}} \int_{0}^{x} g(s, y) d s d y\right. \\
& \left.+\sum_{j=1}^{n} \sum_{k=1}^{m} \frac{\alpha_{j}}{\alpha} \frac{\beta_{k}}{\beta} \int_{0}^{x_{j}} \int_{0}^{y_{k}} g(x, y) d x d y\right) .
\end{aligned}
$$

Then equation (58) in the operator form looks as follows:

$$
(I+A) g+B g=f .
$$

By Lemma 1.4, the operator $I+A$ is invertible in the space $C(D)$ and

$$
\left\|(I+A)^{-1}\right\| \leq e^{9 \gamma} .
$$

On the other hand, the operator $B$ in the space $C(D)$ clearly admits the following estimate:

$$
\|B\| \leq \bar{a} Y \bar{k}_{2}+\bar{b} X \bar{k}_{1}+3 \bar{c} X Y \bar{k}_{1} \bar{k}_{2} \leq 5 \gamma \bar{k}
$$

Since relation (59) is equivalent to the relation

$$
\left.g+(I+A)^{-1} B g=(I+A)\right)^{-1} f,
$$

the above estimates of operators $(I+A)^{-1}$ and $B$, together with condition (55), guarantee unique solvability of equation (60) for each continuous function $f$. This completes the proof of Theorem 2.1 .

2.4. The Fredholm property of problem $\left(\mathcal{P}_{d}\right)$. Here we study the solvability of problem $\left(\mathcal{P}_{d}\right)$ under conditions that are less restrictive than those in 2.2. We do not require that the diameter of the domain $D$ is small; however, $D$ should continue to be small in one direction. The price we pay for loosening restrictions is that the problem becomes Fredholm rather than uniquely solvable.

2.4.1. Reduction of problem $\left(\mathcal{P}_{d}\right)$ to an integral equation. Here we establish a relation between the Fredholm property of the operator $\mathcal{P}_{d}$ and the Fredholm property of a certain integral operator $\mathcal{C}$ acting in the space $C(D)$. To simplify the exposition we assume that

$$
\sum_{j=1}^{n} \alpha_{j}=1, \quad \sum_{k=1}^{m} \beta_{k}=1 .
$$

This implies that the occurring constants do not depend on $\alpha$ and $\beta$ (cf. the previous section). 
As we have seen earlier, problem

$$
\left\{\begin{array}{l}
\partial_{x} \partial_{y} v(x, y)=g(x, y) \quad \text { in } D \\
\sum_{j=1}^{n} \alpha_{j} v\left(x_{j}, y\right)=\varphi(y) \quad \text { on } I_{y}, \\
\sum_{k=1}^{m} \beta_{k} v\left(x, y_{k}\right)=\psi(x) \quad \text { on } I_{x}
\end{array}\right.
$$

is uniquely solvable in the space $\mathcal{W}$ for arbitrary functions $g \in C(D), \varphi \in C^{1}\left(I_{y}\right), \psi \in$ $C^{1}\left(I_{x}\right)$. If conditions (61) hold, the solution (54) of this problem admits an unexpectedly nice form, which later will simulteneously become useful from various points of view. First we note that the group of summands

$$
F(x, y)-\sum_{j=1}^{n} \alpha_{j} F\left(x_{j}, y\right)-\sum_{k=1}^{m} \beta_{k} F\left(x, y_{k}\right)+\sum_{j=1}^{n} \sum_{k=1}^{m} \alpha_{j} \beta_{k} F\left(x_{j}, y_{k}\right),
$$

in the representation of the solution $v(x, y)$, which is equal to

$$
\begin{aligned}
\int_{0}^{x} \int_{0}^{y} g(s, t) d s d t-\sum_{j=1}^{n} \alpha_{j} \int_{0}^{x_{j}} \int_{0}^{y} g(x, t) d x d t \\
\quad-\sum_{k=1}^{m} \beta_{k} \int_{0}^{y_{k}} \int_{0}^{x} g(s, y) d s d y+\sum_{j=1}^{n} \sum_{k=1}^{m} \alpha_{j} \beta_{k} \int_{0}^{x_{j}} \int_{0}^{y_{k}} g(x, y) d y d x
\end{aligned}
$$

can be written in the form

$$
\begin{aligned}
& \sum_{j=1}^{n} \sum_{k=1}^{m} \alpha_{j} \beta_{k}[ \int_{0}^{x} \int_{0}^{y} g(s, t) d s d t-\int_{0}^{x_{j}} \int_{0}^{y_{k}} g(s, t) d s d t \\
& \quad-\int_{0}^{x_{j}} \int_{y_{k}}^{y} g(s, t) d s d t-\int_{0}^{x_{j}} \int_{0}^{y_{k}} g(s, t) d s d t \\
&\left.\quad-\int_{0}^{y_{k}} \int_{x_{j}}^{x} g(s, t) d s d t+\int_{0}^{x_{j}} \int_{0}^{y_{k}} g(s, t) d s d t\right] \\
&=\sum_{j=1}^{n} \sum_{k=1}^{m} \alpha_{j} \beta_{k} \int_{x_{j}}^{x} \int_{y_{k}}^{y} g(s, t) d s d t .
\end{aligned}
$$

The easiest way to see this is to notice that the expression in the brackets is nothing but

$$
\int_{T} g d s d t-\int_{T_{11}} g d s d t-\int_{T_{12}} g d s d t-\int_{T_{11}} g d s d t-\int_{T_{21}} g d s d t+\int_{T_{11}} g d s d t,
$$

where the domains $T_{k \ell}$ are as shown in Figure 1 and $T=\bigcup_{1 \leq k, \ell \leq 2} T_{k \ell}$.

Together with (54) this yields the following final form for the solution of the model problem (62):

$$
v(x, y)=\sum_{j=1}^{n} \sum_{k=1}^{m} \alpha_{j} \beta_{k} \int_{x_{j}}^{x} \int_{y_{k}}^{y} g(s, t) d s d t+\varphi(y)+\psi(x)-\sum_{k=1}^{m} \beta_{k} \varphi\left(y_{k}\right) .
$$

The explicit formula (63) for the solution of problem (62) easily allows us to establish the following result for a general differential operator

$$
\mathcal{L}=\partial_{x} \partial_{y}+a(x, y) \partial_{x}+b(x, y) \partial_{y}+c(x, y) .
$$




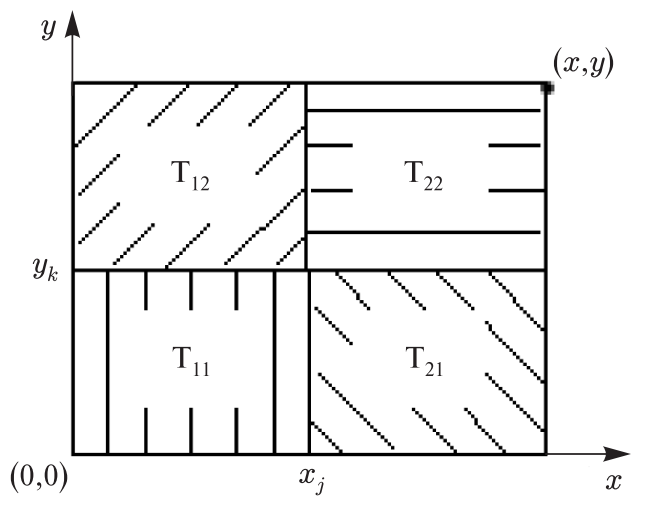

FIGURE 1

Lemma 2.2. If a function $v(x, y) \in \mathcal{W}$ is a solution of problem (62), then the same function satisfies the differential equation

$$
\mathcal{L} v=f(x, y)+a(x, y) \psi^{\prime}(x)+b(x, y) \varphi^{\prime}(y)+c(x, y)\left(\varphi(y)+\psi(x)-\sum \beta_{k} \varphi\left(y_{k}\right)\right),
$$

where

$$
\begin{aligned}
f(x, y)= & g(x, y)+a(x, y) \sum_{k=1}^{m} \beta_{k} \int_{y_{k}}^{y} g(x, t) d t \\
& +b(x, y) \sum_{j=1}^{n} \alpha_{j} \int_{x_{j}}^{x} g(s, y) d s+c(x, y) \sum_{j=1}^{n} \sum_{k=1}^{m} \alpha_{j} \beta_{k} \int_{x_{j}}^{x} \int_{y_{k}}^{y} g(s, t) d s d t .
\end{aligned}
$$

Proof. It is clear that if a function $v(x, y)$ is determined by relation (63), then, as follows from (61), we have

$$
\partial_{x} \partial_{y} v(x, y)=\sum_{j=1}^{n} \sum_{k=1}^{m} \alpha_{j} \beta_{k} g(x, y)=g(x, y) .
$$

Next, differentiating formula (63) we find that

$$
\partial_{x} v(x, y)=\sum_{j=1}^{n} \alpha_{j}\left(\sum_{k=1}^{m} \beta_{k} \int_{y_{k}}^{y} g(x, t) d t\right)+\psi^{\prime}(x)=\sum_{k=1}^{m} \beta_{k} \int_{y_{k}}^{y} g(x, t) d t+\psi^{\prime}(x)
$$

and, similarly,

$$
\partial_{y} v(x, y)=\sum_{j=1}^{n} \alpha_{j} \int_{x_{j}}^{x} g(s, y) d s+\varphi^{\prime}(y) .
$$

The lemma follows immediately.

The integral operator that appears in Lemma 2.2 will play an important role later in the paper, and, therefore, deserves a special notation:

$$
\begin{aligned}
\mathcal{C} g:= & g(x, y)+a(x, y) \sum_{k=1}^{m} \beta_{k} \int_{y_{k}}^{y} g(x, t) d t+b(x, y) \sum_{j=1}^{n} \alpha_{j} \int_{x_{j}}^{x} g(s, y) d s \\
& +c(x, y) \sum_{j=1}^{n} \sum_{k=1}^{m} \alpha_{j} \beta_{k} \int_{x_{j}}^{x} \int_{y_{k}}^{y} g(s, t) d s d t .
\end{aligned}
$$


Moreover, it will be convenient to use the following definition.

Definition. An operator $\mathcal{C}$ is called reciprocal for the differential operator $\mathcal{L}$ with respect to problem $\left(\mathcal{P}_{d}\right)$.

Also, we introduce the following notation:

$$
\ell(\varphi(y), \psi(x))=a(x, y) \varphi^{\prime}(y)+b(x, y) \psi^{\prime}(x)+c(x, y)\left(\varphi(y)+\psi(x)-\sum_{k=1}^{m} \beta_{k} \varphi\left(y_{k}\right)\right) .
$$

The next result characterizes the deep connection between the operators $\mathcal{P}_{d}$ and $\mathcal{C}$.

Lemma 2.3. The problem

$$
\begin{cases}\mathcal{L} u=f & \text { in } D, \\ \sum_{j=1}^{n} \alpha_{j} u\left(x_{j}, y\right)=\varphi(y) & \text { in } I_{y}, \\ \sum_{k=1}^{m} \beta_{k} u\left(x, y_{k}\right)=\psi(x), & \text { in } I_{x}\end{cases}
$$

is solvable for those and only those triples $(f(x, y), \varphi(y), \psi(x))$ for which the equation

$$
\mathcal{C} g=f(x, y)-\ell(\varphi(y), \psi(x))
$$

has at least one solution $g$.

Proof. Let $u(x, y)$ be a solution of problem $\left(\mathcal{P}_{d}\right)$. Introduce the function $g(x, y)=$ $\partial_{x} \partial_{y} u(x, y)$. Since $u(x, y)$ can be viewed as a solution of problem (62), we can apply Lemma 2.2 and find that

$$
\mathcal{L} u=\mathcal{C} g+\ell(\varphi(y), \psi(x)),
$$

so that

$$
\mathcal{C} g=f-\ell(\varphi, \psi) .
$$

In other words, the function $g$ satisfies the required integral equation.

Conversely, for some triple

$$
(f(x, y), \varphi(y), \psi(x))
$$

let $g(x, y)$ be a function that solves the integral equation

$$
\mathcal{C} g=f(x, y)-\ell(\varphi(y), \psi(x)) .
$$

Consider the function $u(x, y) \in \mathcal{W}$ that is a unique solution of problem (62). By Lemma 2.2. this function satisfies the equation

$$
\mathcal{L} u=\mathcal{C} g+\ell(\varphi, \psi),
$$

hence also the equation $\mathcal{L} u=f$. Moreover, by definition, the function $u(x, y)$ is appropriately related to the given functions $\varphi$ and $\psi$. Lemma 2.3 is proved.

The next two lemmas reduce the Fredholm property of problem $\left(\mathcal{P}_{d}\right)$ to a similar question about the operator $\mathcal{C}$.

Lemma 2.4. The relations $\operatorname{dim} \operatorname{ker} \mathcal{P}_{d} \leq \operatorname{dim} \operatorname{ker} \mathcal{C}$ and $\operatorname{dim} \operatorname{coker} \mathcal{P}_{d} \leq \operatorname{dim} \operatorname{coker} \mathcal{C}$ hold.

Proof. Let the functions $u_{1}(x, y), \ldots, u_{N}(x, y)$ form a basis in the kernel of problem $\left(\mathcal{P}_{d}\right)$. Introduce the functions $w_{k}(x, y)=\partial_{x} \partial_{y} u_{k}(x, y), 1 \leq k \leq N$. Using Lemma 2.3 and taking into account that $\varphi=0, \psi=0$, we find that the relation

$$
\mathcal{L} u_{k}=\mathcal{C} w_{k}+\ell(\varphi, \psi)=\mathcal{C} w_{k}
$$


holds for all $k=1, \ldots, N$. Therefore, $w_{k} \in \operatorname{ker} \mathcal{C}$ for the same values of $k$. Let us prove that the functions $w_{k}(x, y)$ are linearly independent. Let us assume that for a nonzero $n$-tuple of numbers $\lambda_{1}, \ldots, \lambda_{N}$ we have

$$
\lambda_{1} w_{1}(x, y)+\cdots+\lambda_{N} w_{N}(x, y)=0
$$

for all $(x, y) \in D$. Introduce the function

$$
U(x, y)=\sum_{k=1}^{N} \lambda_{k} u_{k}(x, y) .
$$

It is clear that this function satisfies the following conditions:

$$
\begin{aligned}
& \partial_{x} \partial_{y} U(x, y)=0, \quad(x, y) \in D, \\
& \sum_{j=1}^{n} \alpha_{j} U(x, y)=0, \quad(x, y) \in I_{y}, \\
& \sum_{k=1}^{m} \beta_{k} U(x, y)=0, \quad(x, y) \in I_{x} .
\end{aligned}
$$

Therefore, by (53), $U(x, y) \equiv 0$. In other words, the functions $u_{k}(x, y), 1 \leq k \leq N$, are linearly dependent, contradicting the assumption that they form a basis.

To prove the second inequality in the lemma let us assume that a triple $Z=(f(x, y)$, $\varphi(y), \psi(x))$ does not belong to the range $\mathcal{R}\left(\mathcal{P}_{d}\right)$ of the operator $\mathcal{P}_{d}$. Then, by Lemma 2.3. the function

$$
G_{Z}(x, y)=f(x, y)-\ell(\varphi(y), \psi(x))
$$

does not belong to the range of the operator $\mathcal{C}$. If the triples $Z_{1}, \ldots, Z_{N}$ are linearly independent modulo the subspace $\mathcal{R}\left(\mathcal{P}_{d}\right)$ or, in other words, for each nonzero $N$-tuple $\lambda_{1}, \ldots, \lambda_{N}$ we have

$$
\lambda_{1} Z_{1}+\cdots+\lambda_{N} Z_{N} \notin \mathcal{R}\left(\mathcal{P}_{d}\right),
$$

then, according to Lemma 2.3 again,

$$
\lambda_{1} G_{Z_{1}}+\cdots+\lambda_{N} G_{Z_{N}} \notin \mathcal{R}(\mathcal{C}),
$$

because all operations we use are linear. This obviously means that

$$
\operatorname{dim} \text { coker } \mathcal{P}_{d} \leq \operatorname{dim} \operatorname{coker} \mathcal{C}
$$

and Lemma 2.4 is proved.

Lemma 2.5. If the range $\mathcal{R}(\mathcal{C})$ of the operator $\mathcal{C}$ is closed, then the range $\mathcal{R}\left(\mathcal{P}_{d}\right)$ of the operator $\mathcal{P}_{d}$ is also closed.

Proof. Let

$$
\mathcal{P}_{d} u_{n}(x, y)=\left(f_{n}(x, y), \varphi_{n}(x), \psi_{n}(y)\right), \quad n=1,2, \ldots,
$$

and suppose the sequence $\left(f_{n}, \varphi_{n}, \psi_{n}\right)$ converges to $(f(x, y), \varphi(x), \psi(y))$ in the space $\mathcal{V}$ as $n \rightarrow \infty$. Then the sequence of functions

$$
F_{n}(x, y)=f_{n}(x, y)-\ell\left(\varphi_{n}(x), \psi_{n}(y)\right)
$$

converges to some function $F(x, y)$ in the space $C(D)$. Since the range $\mathcal{R}(\mathcal{C})$ is closed, there exists a function $w(x, y)$ such that

$$
\mathcal{C} w=F .
$$

By Lemma 2.3, this implies that problem $\mathcal{P}_{d} u=(f, \varphi, \psi)$ is also solvable, thus proving the lemma.

The two latter results imply the following theorem. 
Theorem 2.6. If the operator $\mathcal{C}$ is Fredholm, then the operator $\mathcal{P}_{d}$ is also Fredholm, each in its own pair of spaces. Moreover, if the operator $\mathcal{C}$ is invertible, then the operator $\mathcal{P}_{d}$ is also invertible.

2.4.2. The Fredholm property of the operator $\mathcal{C}$. In this subsection we find conditions for the operator $\mathcal{C}$ to be Fredholm in terms of the constants determining the operator $\mathcal{P}_{d}$. Together with Theorem 2.6 this concludes the analysis of the Fredholm property of problem $\mathcal{P}_{d}$. Let us represent the operator $\mathcal{C}$ as the sum of two operators,

$$
\mathcal{C}=\mathcal{T}+\mathcal{K},
$$

where

$$
\mathcal{T}: g(x, y) \rightarrow g(x, y)+a(x, y) \sum_{k=1}^{m} \beta_{k} \int_{y_{k}}^{y} g(x, t) d t+b(x, y) \sum_{j=1}^{n} \alpha_{j} \int_{x_{j}}^{x} g(s, y) d s
$$

and

$$
\mathcal{K}: g(x, y) \rightarrow c(x, y) \sum_{j=1}^{n} \sum_{k=1}^{m} \alpha_{j} \beta_{k} \int_{x_{j}}^{x} \int_{y_{k}}^{y} g(s, t) d s d t .
$$

Definition. The operator $\mathcal{T}$ is called the principal part of the operator $\mathcal{C}$.

It is clear that the operator $\mathcal{K}$ is compact in the space $C(D)$. Indeed, for each function $g(x, y)$ in the unit sphere of the space $C(D)$, the set of functions $|\operatorname{grad} \mathcal{K} g|$ is uniformly bounded, and the statement follows from the Arcela compactness criterion. Therefore, by the Riesz-Schauder theorem, to establish that the operator $\mathcal{C}$ is Fredholm it suffices to verify that the operator $\mathcal{T}$ is invertible.

First let us assume that $b(x, y)=0$, so that the principal part of the operator $\mathcal{C}$ takes the form

$$
\mathcal{T} g=g(x, y)+a(x, y) \sum_{k=1}^{m} \beta_{k} \int_{y_{k}}^{y} g(x, t) d t, \quad g \in C(\bar{D}) .
$$

Definition. For two sets $\left\{\beta_{k}\right\}_{1}^{m}$ and $\left\{y_{k}\right\}_{1}^{m}, y_{k} \in I_{y}$, of real numbers, we denote by $\Gamma_{\beta}$ the linear functional on the space $C\left(I_{y}\right)$ given by

$$
\Gamma_{\beta}: f(y) \rightarrow \sum_{k=1}^{m} \beta_{k} f\left(y_{k}\right) .
$$

Lemma 2.7. If

$$
\Gamma_{\beta}\left(\exp \left\{-\int_{0}^{y} a(x, t) d t\right\}\right) \neq 0
$$

for all $x$, then the operator $\mathcal{T}$ is invertible in the space $C(D)$.

Proof. Since in the definition of the operator $\mathcal{T}$ the variable $x$ plays the role of an unessential parameter, it can be dropped to simplify the notation. Therefore, we have to prove that the equation

$$
g(z)+a(z) \sum_{k=1}^{m} \beta_{k} \int_{z_{k}}^{z} g(t) d t=h(z)
$$

is uniquely solvable for each function $h(z) \in C\left(I_{y}\right)$. Introduce the new unknown function

$$
G(z)=\int_{0}^{z} g(t) d t
$$


satisfying the condition $G(0)=0$. Then the new equation

$$
G^{\prime}(z)+a(z) \sum_{k=1}^{m} \beta_{k}\left[G(z)-G\left(z_{k}\right)\right]=h(z)
$$

is equivalent to equation (65) and can be written in the form

$$
G^{\prime}(z)+a(z) G(z)-a(z) \Gamma_{\beta}(G)=h(z) .
$$

To solve this equation, we compute a priori the constant $\Gamma_{\beta}(G)$, after which the unique solvability becomes clear. Multiplying both parts of equation (66) by $\exp \left\{\int_{0}^{z} a(t) d t\right\}$ and integrating from 0 to $z$, we find that

$$
G(z) \exp \left\{\int_{0}^{z} a(t) d t\right\}=\Gamma_{\beta}(G) \int_{0}^{z} a(t) \exp \left\{\int_{0}^{t} a(s) d s\right\} d t+h_{1}(z)
$$

where

$$
h_{1}(z)=\int_{0}^{z} h(t) \exp \left\{\int_{0}^{t} a(s) d s\right\} d t .
$$

Computing the integral in the right-hand side of the equation for $G$, we find that

$$
G(z)=\left(\Gamma_{\beta} G\right)\left[1-\exp \left\{-\int_{0}^{z} a(s) d s\right\}\right]+\widetilde{h}(z),
$$

where

$$
\widetilde{h}(z)=h_{1}(z) \exp \left\{-\int_{0}^{z} a(s) d s\right\} .
$$

Applying to both parts of this equality the functional $\Gamma_{\beta}$ we find that

$$
\Gamma_{\beta}(G) \Gamma_{\beta}\left(\exp \left\{-\int_{0}^{z} a(s) d s\right\}\right)=\Gamma_{\beta}(\widetilde{h}) .
$$

Condition (64) allows us to determine the constant $\Gamma_{\beta}(G)$ a priori, after which the unique solvability of equation (66) becomes obvious.

Now let us assume that in problem $\left(\mathcal{P}_{d}\right)$ we have $b(x, y)=b(x)$. Then in the corresponding differential equation $\mathcal{L} u=f$ we replace $u(x, y)$ with the unknown function

$$
u(x, y) \exp \left\{\int_{0}^{x} b(t) d t\right\} \text {. }
$$

Then problem $\left(\mathcal{P}_{d}\right)$ transforms into the equivalent problem

$$
\left\{\begin{array}{l}
\partial_{x} \partial_{y} u+a \partial_{x} u+(c-a b) u=f \exp \left\{\int_{0}^{x} b(t) d t\right\} \\
\sum_{j=1}^{n} \alpha_{j} \exp \left\{-\int_{0}^{x_{j}} b(t) d t\right\} u\left(x_{j}, y\right)=\varphi(y) \\
\sum_{k=1}^{m} \beta_{k} u\left(x, y_{k}\right)=\psi(x) \exp \left\{-\int_{0}^{x} b(t) d t\right\}
\end{array}\right.
$$

to which we can apply Lemma 2.7. As a result, we arrive at the following lemma.

Lemma 2.8. If

$$
\Gamma_{\alpha}\left(\exp \left\{-\int_{0}^{x} b(s) d s\right\}\right) \neq 0 \quad \text { and } \quad \Gamma_{\beta}\left(\exp \left\{-\int_{0}^{y} a(x, t) d t\right\}\right) \neq 0
$$

for all $x$, then the operator $\mathcal{T}$ corresponding to our differential operator $\mathcal{L}$ is invertible. 
2.4.3. The main result. In this subsection we formulate and prove the main result pertaining to the nonlocal problem $\left(\mathcal{P}_{d}\right)$ for a differential operator $\mathcal{L}$ of general form.

Theorem 2.9. Consider problem $\left(\mathcal{P}_{d}\right)$ with the operator

$$
\mathcal{L} u=\partial_{x} \partial_{y} u+a(x, y) \partial_{x} u+b(x, y) \partial_{y} u+c(x, y)
$$

and constants $\left\{\alpha_{j}\right\}_{1}^{n},\left\{\beta_{k}\right\}_{1}^{m}$ satisfying the conditions

$$
\begin{gathered}
\left(\sum_{j=1}^{n} \alpha_{j}\right)\left(\sum_{k=1}^{m} \beta_{k}\right) \neq 0, \quad \Gamma_{\alpha}\left(\exp \left\{-\int_{0}^{x} b(s, 0) d s\right\}\right) \neq 0 \\
\Gamma_{\beta}\left(\exp \left\{-\int_{0}^{y} a(x, t) d t\right\}\right) \neq 0 \quad \text { for all } x
\end{gathered}
$$

Let

$$
w_{y}(b)=\max _{x}|b(x, y)-b(x, 0)|
$$

If

$$
\overline{w_{y}(b)} X
$$

is sufficiently small, then the corresponding operator $\mathcal{P}_{d}$ is Fredholm.

Proof. Let us represent the operator $\mathcal{L}$ in the form

$$
\mathcal{L}=\mathcal{L}_{0}+\Delta \mathcal{L},
$$

where $\mathcal{L}_{0}$ differs from $\mathcal{L}$ only by the coefficient at $\partial_{y}$ : in $\mathcal{L}_{0}$ this coefficient equals $b(x, 0)$. The corresponding operators $\mathcal{C}$ and $\mathcal{C}_{0}$ reciprocal to $\mathcal{L}$ and $\mathcal{L}_{0}$ respectively differ by the operator

$$
g(x, y) \mapsto(b(x, y)-b(0, y)) \int_{0}^{x} g(x, t) d t
$$

whose norm in the space $C(D)$ does not exceed

$$
\overline{w_{y}(b)} X \text {. }
$$

It is clear that the corresponding principal parts $\mathcal{T}$ and $\mathcal{T}_{0}$ of these operators differ by exactly the same operator. Since the invertibility of the operator $\mathcal{T}_{0}$ was proved earlier (Lemma 2.8), the operator $\mathcal{T}$ can be represented in the form

$$
\mathcal{T}=\mathcal{T}_{0}+\left(\mathcal{T}-\mathcal{T}_{0}\right)=\mathcal{T}_{0}\left(I+\mathcal{T}_{0}^{-1}\left(\mathcal{T}-\mathcal{T}_{0}\right)\right)
$$

This immediately implies that if the condition

$$
\overline{w_{y}(b)} X<\left\|\mathcal{T}_{0}^{-1}\right\|
$$

is satisfied, the operator $\mathcal{T}$ is invertible in the space $C(D)$. Therefore, the operator $\mathcal{C}$ reciprocal to the operator $\mathcal{L}$ with respect to problem $\left(\mathcal{P}_{d}\right)$ is Fredholm. By Lemma 2.4 and Lemma 2.5 the same is true for the operator $\mathcal{P}_{d}$.

This concludes the proof of Theorem 2.9. 


\section{REFERENCES}

[1] A. L. Skubachevsky, Elliptic problems with nonlocal boundary conditions. Math. Sbornik 129 (1986), no. 2, 279-302; English transl, Math. USSR-Sb. 57 (1987), no. 1, 279-302. MR832122 (87h:35089)

[2] L. A. Muravei and A. V. Filinovskii, On the non-local boundary value problems for a parabolic equation. Math. Notes 54 (1993), 1045-1057. MR.1256610 (94m:35133)

[3] P. Paneah, Nonlocal problems for linear second order hyperbolic differential equations. Ph. D. Thesis, Technion, Israel, 2005.

TeChnion, Haifa, IsRael

E-mail address: peter@tx.technion.ac.il

Technion, Haifa, IsRael

E-mail address: peter@mellanox.co.il

Translated by O. A. KHLEBORODOVA 